\title{
Dimensional Renormalization and the Action Principle
}

\author{
P. Breitenlohner and D. Maison* \\ Max-Planck-Institut für Physik und Astrophysik, D-8000 München 40, Federal Republic of Germany
}

\begin{abstract}
Dimensional renormalization is defined in such a way that the renormalized action principle holds. It is shown that this leads to a minimal, additive renormalization. The derivation of Ward-Takahashi indentities and Callan-Symanzik equations from the action principle is exemplified.
\end{abstract}

\section{Introduction}

Dimensional regularization $[1,2]$ has become an almost indispensable tool to the perturbative treatment of non-abelian gauge theories. Nevertheless the first complete proof of the so-called Slavnov-Taylor identities, guaranteeing the unitarity of the $S$-matrix, has been given recently with help of the "Normal Product Algorithm" (NPA) based on BPHZ renormalization [3]. The reason lies in the validity of the renormalized action principle in that formalism. The action principle specifies the change of the Green's functions under an infinitesimal variation of fields or parameters entering the Lagrangian. In our opinion it provides a much more efficient approach to the proof of Ward-Takahashi or Slavnov-Taylor identities-or other structural properties-than the algebraic manipulations performed on individual diagrams advocated by the pioneers in that field [4]. The approach of Ref. [3] suffers, however, from the fact that the subtraction method in the BPHZ renormalization is not compatible with the identities to be proved. This is overcome by appropriately chosen asymmetric finite counterterms added to the Lagrangian. Apart from the difficulty to prove that such counterterms can really be found in all orders of perturbation theory the method appears much too clumsy for practical calculation.

Therefore, it is only natural to make an effort to establish the renormalized action principle in the framework of dimensional renormalization. This is one of the main objectives of the present work. The second is to show that dimensional renormalization meets the requirements of a minimal, additive renormalization

* Present address: II. Institut für Theoretische Physik der Universität Hamburg, Luruper Chaussee 149, D-2000 Hamburg 50, Federal Republic of Germany 
in the sense of Hepp [5]. Although there exists already a proof for that by E. R. Speer [6], who reduces dimensional renormalization to standard BPH renormalization (= minimal Taylor series subtractions) performing a suitable finite renormalization, we have included a direct proof without recourse to BPH renormalization. In fact our subtractions differ by finite terms from those proposed by Speer, which proves to be necessary to establish the renormalized action principle and only the latter guarantees the validity of Ward identities etc. ${ }^{1}$

Our proof of the renormalized action principle proceeds in two steps:

i) We demonstrate its validity in the regularized theory. The essential property to show is that the application of the kinetic differential operator to the propagator corresponding to some line $\ell$ in a Feynman graph (i.e. $\square+m^{2}$ for a scalar line) is equivalent to the contraction of that line to a point.

ii) We prove that the subtractions corresponding to all renormalization parts (= superficially divergent 1 PI subgraphs) before and after contraction of the line are identical, although the respective classes of renormalization parts are generally different.

We have decided to devote some space especially to the latter point, since there does not seem to exist an adequate discussion of that problem in the literature $^{2}$. As far as the treatment of covariants in the dimensionally regularized theory is concerned, especially the notorious $\gamma_{5}$, we follow essentially t'Hooft and Veltman [1], with somewhat more emphasis on a consistent formalism. We try to convince the reader that there is no better choice for the definition of $\gamma_{5}$.

In Appendix A we have collected all relevant definitions and properties concerning Feynman graphs.

\section{Dimensional Regularization}

\section{II.1. Feynman Amplitudes}

Assigning propagators $\Delta_{\ell}$ to lines $\ell$ and vertex parts $\hat{X}_{i}$ to vertices $V_{i}$ of some Feynman graph $G$ we may construct Feynman amplitudes $\mathscr{T}_{G}$ as formal expressions of the type ${ }^{3}$ (Definitions are given in Appendix A)

$$
\mathscr{T}_{G}\left(x_{1}, \ldots, x_{M}\right)=\prod_{i \in \mathscr{V}_{G}} \hat{X}_{i} \prod_{\ell \in \mathscr{L}_{G}} \Delta_{\ell}\left(\sum_{i \in \mathscr{V}_{G}} e_{\ell i} x_{i}\right), \quad x_{i} \in \mathbb{R}^{n}, \quad n \text { pos. integer }
$$

with

$$
\begin{aligned}
\hbar^{-1} \Delta_{\ell}(x)= & \lim _{\varepsilon \rightarrow 0} Z_{\ell}(-i \partial / \partial u) \int_{0}^{\infty} d \alpha(-2 i \alpha)^{-n / 2} \\
& \left.\cdot \exp \left\{-(i / 4 \alpha)(x+u)^{2}-i \alpha\left(m_{\ell}^{2}-i \varepsilon\right)\right\}\right|_{u=0} .
\end{aligned}
$$

$Z_{\ell}(-i \partial / \partial u)$ are some constant coefficient diff. operators containing in general covariant algebraic objects like $\gamma$-matrices etc. and $\hbar \hat{X}_{i}=X_{i}\left(-i \partial / \partial x_{i}\right)$ are again constant coeff. diff. operators like the $Z_{\ell}$.

1 A related work by Collins [7] has recently appeared

2 The proof of Y.M.P. Lam Ref. [8] is not complete in our mind

3 The $\hat{X}_{i}$ and $\Delta_{\ell}$ have to be appropriately ordered, which is understood to be done whenever necessary 
Taking the $n \times M$-dimensional Fourier transform of $\mathscr{T}_{G}(x)$ and performing the Gaussian integrations over $x_{i}$ we obtain ${ }^{4}$

$$
\begin{aligned}
\mathscr{T}_{G}\left(p_{1}, \ldots, p_{M}\right)= & (2 \pi)^{-n M / 2} \int e^{i p x} \mathscr{T}_{G}(x) d x \\
= & \left.\lim _{\varepsilon \rightarrow 0} \hbar^{h_{G}-1}(2 \pi)^{n / 2} \delta\left(\sum p_{i}\right) i^{h_{G}}(2 i)^{-n h_{G} / 2} \int_{0}^{\infty} \prod_{\ell \in \mathscr{L}_{G}} d \alpha_{\ell} I_{\varepsilon}(p, \underline{u}, \underline{\alpha})\right|_{u=0} ; \\
I_{\varepsilon}(p, \underline{u}, \underline{\alpha})= & d(\alpha)^{-n / 2} \prod_{i \in \mathscr{V}_{G}} X_{i}\left(p_{i},-i \partial / \partial u_{\ell}\right) \prod_{\ell \in \mathscr{L}_{G}} Z_{\ell}\left(-i \partial / \partial u_{\ell}\right) \exp i W(p, \underline{u}, \underline{\alpha}) ; \\
W(p, \underline{u}, \underline{\alpha})= & \left(p^{+}-u^{+}(2 \alpha)^{-1} e_{E}, u^{+}\right)\left(\begin{array}{cc}
A_{E}^{-1} & 0 \\
0 & -(4 \alpha)^{-1}
\end{array}\right)\left(\begin{array}{c}
p-e_{E}^{+}(2 \alpha)^{-1} u \\
u
\end{array}\right) \\
& -\sum_{\ell \in \mathscr{L}_{G}} \alpha_{\ell}\left(m_{\ell}^{2}-i \varepsilon\right) \\
= & \left(p^{+}, u^{+}\right)\left(\begin{array}{cc}
0 & -2 e_{E}^{+} \\
-2 e_{E} & -4 \alpha
\end{array}\right)^{-1}\left(\begin{array}{l}
p \\
u
\end{array}\right)-\sum_{\ell \in \mathscr{L}_{G}} \alpha_{\ell}\left(m_{\ell}^{2}-i \varepsilon\right) .
\end{aligned}
$$

This expression is the starting point for the dimensional regularization. It is achieved in two steps:

i) we interpret the parameter $n$ appearing in (3) as a complex variable,

ii) we give a definition of the algebraic objects (like $\gamma$-matrices, momentum 4-vectors etc.) appearing in (3) which makes no direct use of the concept of dimension (see Sect. II.2).

\section{II.2. " $n$-Dimensional" Lorentz Covariants}

For the validity of canonical Ward-Takahashi identities etc. for the dimensionally regularized Feynman amplitudes resp. Green's functions it is essential to find an extension of the Lorentz covariants $\left(\gamma_{\mu}, p_{\mu}, g_{\mu \nu}\right.$ etc.) which allows one to manipulate these objects like in 4-dimensions. This will be achieved by treating them as formal objects obeying certain algebraic identities. We shall make no use of the fact that indices can assume values, but treat objects like $\gamma_{\mu}$ as just one entity (i.e. we shall never use $\gamma_{2}$ etc.). The only place where one runs into troubles with that prescription is where the $\varepsilon_{\kappa \lambda \mu \nu}$-tensor comes into play, since it is a genuine 4-dimensional object (like $\varepsilon_{i j k}$ is a 3 -dimensional one). This is expressed by its property that in 4 dimensions the value of an arbitrary Lorentz index is equal to exactly one of the values of the indices of $\varepsilon$. Correspondingly there are identities involving $\varepsilon$ depending crucially on 4-dimensionality as

$$
\varepsilon_{\mu_{1} \ldots \mu_{4}} \varepsilon_{v_{1} \ldots v_{4}}=-\sum_{\pi \in S_{4}} \operatorname{sign} \pi \prod_{i=1}^{4} g_{\mu_{i} v_{\pi(i)}}
$$

and as a consequence

$$
\sum_{\pi \in S_{5}} \operatorname{sign} \pi \varepsilon_{\mu_{\pi(1)} \ldots \mu_{\pi(4)}} g_{\mu_{\pi(5)}}=0 .
$$

$4 \quad$ Using the relation $\left[p_{i}+e_{i \ell}^{+}\left(-i \partial / \partial u_{\ell}\right)\right] I_{\varepsilon}(p, \underline{u}, \underline{\alpha})=0$ the vertex factors $X_{i}\left(p_{i}\right)$ can be included in the factors $Z_{\ell}\left(-i \partial / \partial u_{\ell}\right)$ 
Two possibilities are to be considered:

i) no use is made of (4), i.e. there is no possibility to reduce a product of several $\varepsilon$-tensors in the formal algebra,

ii) in addition to the " $n$-dimensional" covariants also "( $n-4)$-dimensional" ones (or equivalently 4-dimensional ones) are introduced.

Possibility ii) allows a further simplification of covariants containg a product of several $\varepsilon$-tensors; as a consequence $\gamma_{5}^{2}=-1$ can be proved.

Equation (5), however, has the disadvantage that it prevents one from finding easily a unique normal form for the covariants (see below).

We use the usual symbols ${ }^{5} g_{\mu \nu}, p_{\mu}, \gamma_{\mu}, \mathbb{1}, \varepsilon_{\kappa \lambda \mu \nu}$ which can be added, multiplied etc. as usual, obeying the additional identities ${ }^{6}$ (all symbols except $\gamma_{\mu}$ are assumed to commute)

$$
\begin{aligned}
& g_{\mu \nu} g_{\nu \lambda}=g_{\mu \lambda}, \quad g_{\mu \nu}=g_{\nu \mu}, \\
& g_{\mu \nu} p_{\nu}=p_{\mu}, \quad g_{\mu \nu} \gamma_{\nu}=\gamma_{\mu}, \quad p_{\mu} q_{\mu}=p \cdot q, \\
& g_{\mu \nu} \varepsilon_{v \varrho \sigma \tau}=\varepsilon_{\mu \varrho \sigma \tau}, \\
& \left\{\gamma_{\mu}, \gamma_{\nu}\right\}=\gamma_{\mu} \gamma_{\nu}+\gamma_{\nu} \gamma_{\mu}=2 g_{\mu \nu} \mathbb{1}, \quad \mathbb{1} \gamma_{\mu}=\gamma_{\mu} \mathbb{1}=\gamma_{\mu}, \\
& g_{\mu \mu}=n, \\
& \operatorname{Tr} \mathbb{1}=4 .
\end{aligned}
$$

In order to be able to formulate Equation (4) we introduce additional symbols (as discussed above) $\hat{g}_{\mu \nu}, \hat{p}_{\mu}, \hat{\gamma}_{\mu}$ obeying the identities

$$
\begin{aligned}
& g_{\mu \nu} \hat{g}_{\nu \lambda}=\hat{g}_{\mu \nu} \hat{g}_{\nu \lambda}=\hat{g}_{\mu \lambda}, \quad \hat{g}_{\mu \nu}=\hat{g}_{v \mu}, \\
& \hat{g}_{\mu \nu} p_{\nu}=\hat{p}_{v}, \quad \hat{g}_{\mu \nu} \gamma_{\nu}=\hat{\gamma}_{\mu} ; \\
& \varepsilon_{\mu_{1} \ldots \mu_{4}} \varepsilon_{v_{1} \ldots v_{4}}=-\sum_{\pi \in S_{4}} \operatorname{sign} \pi \prod_{i=1}^{4}\left(g_{\mu_{i} v_{\pi(2)}}-\hat{g}_{\mu_{i} v_{\pi(i)}}\right)
\end{aligned}
$$

where $S_{4}$ denotes the permutation group of 4 objects. From these assumptions we can prove for example

$$
\begin{aligned}
& g_{\mu \nu} \hat{\gamma}_{\nu}=\hat{g}_{\mu \nu} \hat{\gamma}_{\nu}=\hat{\gamma}_{\mu}, \\
& \left\{\gamma_{\mu}, \hat{\gamma}_{\nu}\right\}=\left\{\hat{\gamma}_{\mu}, \hat{\gamma}_{\nu}\right\}=2 \hat{g}_{\mu \nu} \mathbb{1}, \\
& \varepsilon_{\varkappa \lambda \mu \nu} \hat{g}_{\nu \varrho}=0, \\
& \varepsilon_{\mu_{1} \ldots \mu_{4}}=\operatorname{sign} \pi \varepsilon_{\mu_{\pi(1)} \ldots \mu_{\pi(4)}}, \\
& \hat{g}_{\mu \mu}=n-4, \\
& \operatorname{Tr} \gamma_{\mu}=0 .
\end{aligned}
$$

Before we give a definition for $\gamma_{5}$, we will try to convince the reader, that there is no better choice. In order to do so, we observe, that $\gamma_{5}$ can be defined for $n=4$ by its two properties

$$
\text { i) } \operatorname{Tr}\left(\gamma_{5} \gamma_{\mu_{1}} \ldots \gamma_{\mu_{4}}\right)=\varepsilon_{\mu_{1} \ldots \mu_{4}} \operatorname{Tr} \mathbb{1}
$$

5 This list may be enlarged if necessary by other covariant objects

6 There are a lot of obvious rules to be observed, like: no index $\mu$ can appear more than twice in one product etc., which we do not specify here 
which will be maintained and

ii) $\left\{\gamma_{\mu}, \gamma_{5}\right\}=0$

which has to be given up, because the two are not compatible for $n \neq 4$. As an alternative we might try to maintain ii), but this leads immediately to

$$
n(n-2)(n-4) \operatorname{Tr}\left(\gamma_{5} \gamma_{\mu_{1}} \ldots \gamma_{\mu_{4}}\right)=0
$$

and there is no smooth limit $n \rightarrow 4$ which reproduces i). Nevertheless, this approach strongly supports the result that axial anomalies can always be avoided except in matrix elements where i) becomes essential.

Let us define $\gamma_{5}=(4 !)^{-1} \varepsilon_{\mu_{1} \ldots \mu_{4}} \gamma_{\mu_{1}} \ldots \gamma_{\mu_{4}}$. With that definition we can prove

Proposition 1. $\left\{\gamma_{\alpha}, \gamma_{5}\right\}=\left\{\hat{\gamma}_{\alpha}, \gamma_{5}\right\}=2 \hat{\gamma}_{\alpha} \gamma_{5}$.

Using the abbreviations $\hat{g}_{\mu \nu}=g_{\mu \nu}-\hat{g}_{\mu \nu}, \hat{\gamma}_{\mu}=\gamma_{\mu}-\hat{\gamma}_{\mu}$ etc. we show first

Lemma 1. $\sum_{\pi \in S_{5}} \operatorname{sign} \pi \varepsilon_{\mu_{\pi(1)} \ldots \mu_{\pi(4)}} \hat{\mathrm{g}}_{\mu_{\pi(5) \alpha}}=0$.

Proof. $\sum_{\pi \in S_{5}} \operatorname{sign} \pi \varepsilon_{\mu_{\pi(1)} \ldots \mu_{\pi(4)}} \hat{\mathrm{g}}_{\mu_{\pi(5) \alpha}}$

$$
\begin{aligned}
& =-1 / 6 \sum_{\pi \in S_{S}} \operatorname{sign} \pi \varepsilon_{\left.\mu_{\pi(1)} \ldots \mu_{\pi(4)}\right)} \varepsilon_{\mu_{\pi(5)} v_{1} v_{2} v_{3}} \varepsilon_{\alpha_{\alpha_{1} v_{2} v_{3}}} \\
& =4 \varepsilon_{\alpha v_{1} v_{2} v_{3}} \sum_{\pi \in S_{5}} \operatorname{sign} \pi \hat{\mathrm{g}}_{\left.\mu_{\pi(1)} \mu_{\pi(5)}\right)} \hat{\mathrm{g}}_{\mu_{\pi(2)} v_{1} v_{1}} \hat{\mathrm{g}}_{\mu_{\pi(3)} v_{2}} \hat{\mathrm{g}}_{\mu_{\pi(4)} v_{3}}=0
\end{aligned}
$$

since $\hat{g}_{\mu_{\pi(1)} \mu_{\pi(5)}}$ is symmetric under the exchange of 1 and 5 .

Proof of Proposition 1. Using the above Lemma we get

$$
\begin{aligned}
0= & (1 / 4 !)\left(\gamma_{\mu_{1}} \ldots \gamma_{\mu_{5}}+\gamma_{\mu_{5}} \ldots \gamma_{\mu_{1}}\right) \sum_{\pi \in S_{5}} \operatorname{sign} \pi \varepsilon_{\mu_{\pi(1)} \ldots \mu_{\pi(4)}} \hat{\hat{g}}_{\mu_{\pi(5) \alpha}} \\
= & (5 / 4 !)\left(\hat{\gamma}_{\alpha} \gamma_{\mu_{1}} \ldots \gamma_{\mu_{4}}+\gamma_{\mu_{4}} \ldots \gamma_{\mu_{1}} \hat{\gamma}_{\alpha}\right) \sum_{\pi \in S_{4}} \operatorname{sign} \pi \varepsilon_{\mu_{\pi(1)} \ldots \mu_{\pi(4)}} \\
& +(20 / 3 !) \hat{\hat{g}}_{v \alpha}\left(\gamma_{\mu_{1}} \ldots \gamma_{\mu_{3}}+\gamma_{\mu_{3}} \ldots \gamma_{\mu_{1}}\right) \sum_{\pi \in S_{3}} \operatorname{sign} \pi \varepsilon_{\mu_{\pi(1)} \mu_{\pi(2)} \mu_{\pi(3)} v} \\
= & 5 !\left\{\hat{\hat{\gamma}}_{\alpha}, \gamma_{5}\right\} \quad \text { and hence }\left\{\gamma_{\alpha}, \gamma_{5}\right\}=\left\{\hat{\gamma}_{\alpha}, \gamma_{5}\right\}
\end{aligned}
$$

where use is made of the fact that the permutation (54321) is even, whereas (321) is odd. The second part of Equation (9) is trivial to prove.

From Proposition 1 one can immediately compute

$$
\operatorname{Tr}\left(\left\{\gamma_{\alpha}, \gamma_{5}\right\} \gamma_{\alpha} \gamma_{\lambda} \gamma_{\mu} \gamma_{\nu} \gamma_{\varrho}\right)=8(n-4) \varepsilon_{\lambda \mu \nu e}
$$

which is important for the derivation of the Adler anomaly.

Similarly one may prove

Proposition 2. $\gamma_{5}^{2}=-\mathbb{1}$.

Remark. All the quantities appearing are treated as real, treating charges by doubling the number of components. 
The following proposition can be proved:

The identities above can be used as simplification rules for syntactically correct expressions, leading to a normal form (NF) which is unique insofar as (4) is neglected-otherwise there remain further linear relations with $n$-independent coefficients between expressions in NF derived from Equation (5).

As for the proof we execute the following transformations (simplifications) leading to the NF:

(i) we eliminate $\gamma_{5}$ by its definition;

(ii) we eliminate $\operatorname{Tr}$ by applying $\left\{\gamma_{\mu}, \gamma_{\nu}\right\}=2 g_{\mu \nu}, \operatorname{Tr} \gamma_{\mu}=0, \operatorname{Tr} \mathbb{1}=4, \operatorname{Tr} A B=$ $\operatorname{Tr} B A$;

(iii) we antisymmetrize products of $\gamma$ 's by $\left\{\gamma_{\mu}, \gamma_{\nu}\right\}=2 g_{\mu \nu}$;

[(iv) we eliminate $\varepsilon$-tensors by $\left.\left(4^{\prime}\right)\right]$; etc.;

(v) we eliminate $g_{\mu \nu}$ 's (resp. $\hat{g}_{\mu \nu}$ 's) by $g_{\mu \nu} p_{v}=p_{\mu}$ (resp. $\left.\hat{g}_{\mu \nu} p_{v}=g_{\mu \nu} \hat{p}_{v}=\hat{g}_{\mu \nu} \hat{p}_{\nu}=\hat{p}_{\mu}\right)$

(vi) we replace $p_{\mu} q_{\mu}$ by $p \cdot q$ (resp. $\hat{p}_{\mu} q_{\mu}=p_{\mu} \hat{q}_{\mu}=\hat{p}_{\mu} \hat{q}_{\mu}$ by $\left.\widehat{p \cdot q}\right)$ etc. and use $g_{\mu \mu}=n$ (resp. $\hat{g}_{\mu \mu}=n-4$ ).

It is easy to see, that [without $\left(4^{\prime}\right)$ ] any further application of any of the identities (6) no longer changes the expression obtained after the application of (i) to (vi).

Having given a meaning to the Lorentz covariants contained in Equation (3) we can now come to the definition of the dimensionally regularized Feynman amplitude corresponding to the formal expression (3):

This is done in two steps.

Step 1: Interprete the covariants contained in the $X_{i}$ 's resp. $Z_{\ell}$ 's (i.e. also $u_{\ell}$ and $\left.\partial / \partial u_{\ell}\right)$ as elements of the algebra described above. The exponential function defining $I_{\varepsilon}(p, u, \alpha)$ is considered as a formal power series. Next execute all derivatives $\partial / \partial u_{\ell}$ and put $u_{\ell}=0$. Reduce the expressions obtained to their NF. This displays explicitly the $n$-dependence of the covariants and therefore can be used for the definition of the counterterms (see Sect. III).

Step 2: Once the counterterms are determined, we interprete the covariants in the NF as 4-dimensional (specifically we put all $\hat{\mathcal{O}}=0$, i.e. $\hat{g}_{\mu \nu}=0, \hat{p}_{\mu}=0$ etc.) and set all of the $M-1$ momenta $p_{i}$ to zero which correspond to internal vertices. After that the Feynman integrand can be considered as a distribution in the remaining $M^{\prime}-1$ momenta over $\mathscr{S}\left(\mathbb{R}^{4\left(M^{\prime}-1\right)}\right)$ and depends parametrically on $\underline{\alpha}$ and $n$. Taking $\operatorname{Re} n \ll 0$ all $\alpha$-integrals converge absolutely. For $\min \left\{m_{\ell}\right\} \neq 0$ the limit $\varepsilon \rightarrow 0$ exists in $\mathscr{S}^{\prime}\left(\mathbb{R}^{4\left(M^{\prime}-1\right)}\right)$ and defines a distribution $\mathscr{T}_{G}(p, n)$ over $\mathscr{S}\left(\mathbb{R}^{4 M^{\prime}}\right)$ [9]. $\mathscr{T}_{G, \varepsilon}(p, n)$ can be continued analytically to the whole complex $n$-plane as a meromorphic function, the singularities of which lie on the manifolds

$$
\omega_{H}(n)=n h_{H}-2 L_{H}+\sum_{\mathscr{L}_{\boldsymbol{H}}} r_{\ell}=k, \quad k=0,1,2, \ldots, H \subset G
$$

where $r_{\ell}$ is the degree of $Z_{\ell}$ [10]. For values of $n$ not on any of these manifolds and $\varepsilon \geqq 0 \mathscr{T}_{G, \varepsilon}(p, n)$ is continuous in $\varepsilon$. Therefore, $\mathscr{T}_{G}(p, n)$ is again a meromorphic function of $n$ with singularities on the same manifolds. 


\section{II.3. The Action Principle}

Most of the equations characterizing the structural properties of a Lagrangian QFT like equations of motion, Ward-Takahashi identities, Callan-Symanzik equations etc. can be understood as a consequence of the "action principle" [11]. Let us consider a Lagrangian $\mathscr{L}=\mathscr{L}_{0}=\mathscr{L}_{\text {int }}$ depending on a set of commuting or anticommuting quantized fields genericly called $\phi(x)$, unquantized "external" fields $\underline{a}(x)$ and space-time independent parameters $\underline{\lambda}$. Then we may ask how the Green's functions of the theory change under an infinitesimal variation of these quantities. The answer, given by the quantum action principle, is most efficiently expressed in terms of the generating functional $Z(\underline{a}, \underline{\lambda})$ for the Green's functions ${ }^{7}$ given by the Gell-Mann-Low series

$$
Z(\underline{\alpha}, \underline{\lambda})=\left\langle\exp i \hbar^{-1} \int \mathscr{L}_{\text {int }}(\phi(x), \underline{a}(x), \underline{\lambda}) d x\right\rangle_{+, c}^{0} .
$$

$Z(\underline{a}, \underline{\lambda})$ is a well defined formal power series in $\hbar$ using dimensionally regularized Feynman amplitudes corresponding to the graphical expansion of $\left\langle\exp i \hbar^{-1} \int \mathscr{L}_{\text {int }}\right\rangle_{+, c}^{0}\langle\rangle_{+, c}^{0}$ designates the connected vacuum expectation value of the time ordered product, evaluated in the free theory given by $L_{0}=\frac{1}{2} \phi D \phi$, i.e. with propagator $i \hbar D^{-1}$. About $D$ we make the assumption that it is a real, second order differential operator with constant coefficients, i.e.

$$
\begin{aligned}
& D_{i j}=a_{i j}+b_{i j}^{\mu} \partial_{\mu}+c_{i j}^{\mu \nu} \partial_{\mu} \partial_{v} \\
& a_{i j}= \pm a_{j i}, \quad b_{i j}^{\mu}=\mp b_{j i}^{\mu}, \quad c_{i j}^{\mu \nu}= \pm c_{j i}^{\mu \nu} \quad \text { the } \pm \text { sign referring }
\end{aligned}
$$

to Bose resp. Fermi components of $\phi=\left(\phi_{i}\right)$. The constants $a, b, c$ contain in general algebraic objects like $\gamma_{\mu}, g_{\mu \nu}$ etc. We assume, however, that $D^{-1}$ is the same algebraic expression as in 4 dimensions, i.e. $D\left(-i \partial / \partial u_{\ell}\right) Z_{\ell}\left(-i \partial / \partial u_{\ell}\right)=$ $-\left(\square_{u_{\ell}}+m_{\ell}^{2}\right)$, which can easily be proved for the standard representations like $-\left(\square+m^{2}\right),-i \gamma_{\mu} \partial_{\mu}+m$ etc., using the algebraic rules of Section II.2. form:

The action principle for the respective kinds of variations takes the following

i) variations of the quantized field $\phi(x)$

$\delta \underline{\phi}(x)=\underline{P}(\phi(x)) \delta \varepsilon(x)$ with some polynomial $\underline{P}$ leave $Z(\underline{a}, \underline{\lambda})$ invariant:

$$
0=\left\langle\delta \mathscr{L} \exp i \hbar^{-1} \int \mathscr{L}_{\text {int }}(x) d x\right\rangle_{+, c}^{0}
$$

where $\delta \mathscr{L}$ is the linear part of $\mathscr{L}(\phi+\delta \phi)-\mathscr{L}(\phi)$ in $\delta \varepsilon$. Neglecting ordering of factors we may write

resp.

$$
\begin{aligned}
\delta \mathscr{L}= & \int\left\{(\delta \mathscr{L} / \delta \phi(x)) P(\phi(x))+\left(\delta \mathscr{L} / \delta \partial_{\mu} \phi(x)\right) \partial_{\mu} P(\phi(x))\right. \\
& -\partial_{\mu}\left(\left(\delta \mathscr{L} / \delta \partial_{\mu} \phi(x)\right) P(\phi(x))\right\} \delta \varepsilon(x) d x
\end{aligned}
$$

$$
\delta \mathscr{L}=\int P(\phi(x))\left((\delta \mathscr{L} / \delta \phi(x))-\partial_{\mu}\left(\delta \mathscr{L} / \delta \partial_{\mu} \phi(x)\right)\right) \delta \varepsilon(x) d x ;
$$

ii) variations of external fields result in

$$
-i \hbar \delta Z / \delta \underline{\alpha}(x)=\left\langle(\delta \mathscr{L} / \delta \underline{a}(x)) \exp i \hbar^{-1} \int \mathscr{L}_{\mathrm{int}}(x) d x\right\rangle_{+, c}^{0} ;
$$

\footnotetext{
7 We assume that $\mathscr{L}_{\text {int }}$ contains a "source term" $\int \underline{J}(x) \phi(x) d x$ which allows to express Green's
} functions as functional derivatives of $Z$ 
iii) variations of parameters give

$$
-i \hbar \partial Z / \partial \underline{\lambda}=\left\langle(\partial \mathscr{L} / \partial \underline{\lambda}) \exp i \hbar^{-1} \int \mathscr{L}_{\text {int }}(x) d x\right\rangle_{+, c}^{0} .
$$

In the following we try to show that Equations (13)-(15) hold in the sense of formal power series in $\hbar$ in the dimensionally regularized theory corresponding to $\mathscr{L}$.

The only difficulty of the proof lies in the asymmetrical treatment of $\mathscr{L}_{0}$ and $\mathscr{L}_{\text {int }}$ in the definition of $Z$ via Feynman graphs: $\mathscr{L}_{0}$ determines the lines, $\mathscr{L}_{\text {int }}$ the vertices. We have to demonstrate how the variations in both constituents conspire to give the simple result of Equations (13)-(15). Two kinds of properties are responsible for this conspiracy:

i) algebraic properties of the Feynman integrands $I(p, u, \alpha)$,

ii) combinatorial properties of the graphical expansion of

$$
\left\langle\exp i \hbar^{-1} \int \mathscr{L}_{\text {int }}(x) d x\right\rangle_{+, c}^{0} \text {. }
$$

We shall restrict ourselves to the discussion of point i), since ii) is straightforward and has nothing to do with dimensional regularization. This immediately settles Equation (14), because we have assumed that there is no external field dependence in $\mathscr{L}_{0}$ and therefore we may write Equation (14) in the form

$$
-i \hbar \delta Z / \delta \underline{a}(x)=\left\langle\left(\delta \mathscr{L}_{\text {int }} / \delta \underline{a}(x)\right) \exp _{i \hbar^{-1}} \int \mathscr{L}_{\text {int }}(x) d x\right\rangle_{+, c}^{0}
$$

the proof of which is essentially combinatorial.

Equation (13) needs somewhat more work. Splitting $\mathscr{L}$ into $\mathscr{L}_{0}$ and $\mathscr{L}_{\text {int }}$ we may rewrite it as

$$
\begin{aligned}
& \left\langle P(\phi(x)) D \phi(x) \exp i \hbar^{-1} \int \mathscr{L}_{\text {int }}(x) d x\right\rangle_{+, c}^{0} \\
& =-\left\langle P(\phi(x))\left(\delta \mathscr{L}_{\text {int }} / \delta \phi(x)\right) \exp i \hbar^{-1} \int(x) d x\right\rangle_{+, c}^{0} .
\end{aligned}
$$

Reducing Equation (17) to Feynman graphs it boils down to the following property: replacing $Z_{\ell}\left(-i \partial / \partial u_{\ell}\right)$ in Equation (3) by $D_{\ell}\left(-i \partial / \partial u_{\ell}\right) Z_{\ell}\left(-i \partial / \partial u_{\ell}\right)=$ $-\left(\square_{u_{\ell}}+m_{\ell}^{2}\right)$ is equivalent to the contraction of line $\ell$ to a point, which is achieved by replacing $\int_{0}^{\infty} d \alpha_{\ell}$ by $-\int_{0}^{\infty} d \alpha_{\ell} \partial / \partial \alpha_{\ell}$, i.e. $\mathscr{T}_{G}^{\prime}=\mathscr{T}_{G / \ell}$, where $\mathscr{T}_{G}^{\prime}$ is the Feynman amplitude for $G$ with an additional factor $D_{\ell}\left(-i \partial / \partial u_{\ell}\right)$ included. Therefore, it is sufficient to show

$$
\left(\square_{u_{\ell}}+m_{\ell}^{2}-i \varepsilon\right) I_{\varepsilon}(p, u, \alpha)=i\left(\partial / \partial \alpha_{\ell}\right) I_{\varepsilon}(p, u, \alpha)
$$

in the formal algebra of covariants. Up to trivial factors ${ }^{8} I_{\varepsilon}(p, u, \alpha)$ has the form (setting $\left.\prod_{i \in \mathscr{V}_{G}} X_{i}\left(p_{i},-i \partial / \partial u_{\ell}\right) \prod_{\ell \in \mathscr{L}_{G}} Z_{\ell}\left(-i \partial / \partial u_{\ell}\right)=Z(-i \partial / \partial u)\right)$,
$\quad I_{\varepsilon}(p, u, \alpha)=Z(-i \partial / \partial u) d(\alpha)^{-n / 2} \exp \left\{i V(p, u, \alpha)-i \sum_{\ell \in \mathscr{L}_{G}} \alpha_{\ell}\left(m_{\ell}^{2}-i \varepsilon\right)\right\}$

8 Actually it is important that the only dependence of these factors on the number $L$ of lines resp. $M$ of vertices is through the number of loops $h=L-M+1$, since the latter does not change by contracting a line 
with

$$
V(p, u, \alpha)=\left(p^{+}, u^{+}\right) M^{-1}\left(\begin{array}{l}
p \\
u
\end{array}\right) \quad M=\left(\begin{array}{cc}
0 & -2 e^{+} \\
-2 e & -4 \alpha
\end{array}\right), d(\alpha)=\operatorname{det} M /(-4)^{L} .
$$

Differentiation with respect to $\alpha_{\ell}$ gives

$$
-i \partial I_{\varepsilon} / \partial \alpha_{\ell}=i\left(2^{-1} n d^{-1} \partial d / \partial \alpha_{\ell}-i \partial V / \partial \alpha_{\ell}+i\left(m_{\ell}^{2}-i \varepsilon\right)\right) I_{\varepsilon}
$$

whereas

$$
-\left(\square_{u_{\ell}}+m_{\ell}^{2}-i \varepsilon\right) I_{\varepsilon}=i\left(-\square_{u_{\ell}} V-i\left(\partial V / \partial u_{\ell}\right) \cdot\left(\partial V / \partial u_{\ell}\right)+i\left(m_{\ell}^{2}-i \varepsilon\right)\right) I_{\varepsilon} .
$$

Using

$$
2^{-1} n d^{-1} \partial d / \partial \alpha_{\ell}=2^{-1} n \operatorname{Tr}\left(\left(\partial M / \partial \alpha_{\ell}\right) M^{-1}\right)=-\square_{u_{\ell}} V
$$

(here $g_{\mu \mu}=n$ comes in !) and

$$
\partial V / \partial \alpha_{\ell}=-\left(p^{+} u^{+}\right) M^{-1}\left(\partial M / \partial \alpha_{\ell}\right) M^{-1}\left(\begin{array}{l}
p \\
u
\end{array}\right)=\left(\partial V / \partial u_{\ell}\right) \cdot\left(\partial V / \partial u_{\ell}\right)
$$

we arrive at the desired equality (18).

Now we go over to the corresponding dimensionally regularized Feynman amplitude of both sides of Equation (18) as described above and obtain $\mathscr{T}_{G}^{\prime}(\underline{p}, n)=$ $\mathscr{T}_{G / \ell}(p, n)$ for $\operatorname{Re} n \ll 0$. Analytic continuation and combinatorics will complete the proof of (13).

It remains to prove Equation $(15)^{9}$. Again reducing it to the language of graphs it means

$$
-i \partial \mathscr{T}_{G} / \partial m_{\ell}=\mathscr{T}_{G}^{\prime}
$$

where $G^{\prime}$ is obtained from $G$ by insertion of the 2-vertex with vertex part $\partial D / \partial m_{\ell}$ into line $\ell$ of $G$.

From

$$
\begin{aligned}
& D Z_{\ell}=-\left(\square_{u_{\ell}}+m_{\ell}^{2}\right) \quad \text { we get }\left(\partial D / \partial m_{\ell}\right) Z_{\ell}+D \partial Z_{\ell} / \partial m_{\ell}=-2 m_{\ell} \\
& \text { resp. }\left(\square_{u_{\ell}}+m_{\ell}^{2}\right) \partial Z_{\ell} / \partial m_{\ell}=2 m_{\ell} Z_{\ell}+Z_{\ell}\left(\partial D / \partial m_{\ell}\right) Z_{\ell} .
\end{aligned}
$$

Using Equation (18) and $\int_{0}^{\infty} d \alpha_{\ell} I_{G}(p, u, \alpha)=-\int_{0}^{\infty} d \alpha_{\ell} \alpha_{\ell} \partial I_{G} / \partial \alpha_{\ell}$ we get ${ }^{10}$ for sufficiently small $n$

$$
\begin{aligned}
-i \partial \mathscr{T}_{G} / \partial m_{\ell} & \approx-\left.i \int_{0}^{\infty}\left(\left(\partial Z_{\ell} / \partial m_{\ell}\right) Z_{\ell}^{-1}-2 i \alpha_{\ell} m_{\ell}\right) I_{G}(p, u, \alpha) d \alpha_{\ell}\right|_{u=0} \\
& =\left.\int_{0}^{\infty}\left(i \alpha_{\ell}\left(\partial / \partial \alpha_{\ell}\right)\left(\partial Z_{\ell} / \partial m_{\ell}\right) Z_{\ell}^{-1}-2 \alpha_{\ell} m_{\ell}\right) I_{G} d \alpha_{\ell}\right|_{u=0} \\
& =\left.\int_{0}^{\infty} \alpha_{\ell} Z_{\ell}\left(\partial D_{\ell} / \partial m_{\ell}\right) I_{G} d \alpha_{\ell}\right|_{u=0} .
\end{aligned}
$$

9 The only "nontrivial" case is, when $\mathscr{L}_{0}$ depends on $\lambda$. We consider only the case where $\lambda$ is a mass, but the other possibilities can be treated in a similar way

10 Correct ordering of the $Z$-factors is understood here 
On the other hand

$$
\begin{aligned}
\mathscr{T}_{G^{\prime}} & \left.\approx \int_{0}^{\infty} d \alpha_{\ell} d \alpha_{\ell^{\prime}} Z_{\ell^{\prime}}\left(-i \partial / \partial u_{\ell^{\prime}}\right)\left(\partial D / \partial m_{\ell^{\prime}}\right) I_{G}\left(p, u_{\ell}+u_{\ell^{\prime}}, \alpha_{\ell}+\alpha_{\ell^{\prime}}\right)\right|_{u=0} \\
& =\left.\int_{0}^{\infty} \alpha_{\ell} d \alpha_{\ell} Z_{\ell}\left(-i \partial / \partial u_{\ell}\right)\left(\partial D / \partial m_{\ell}\right) I_{G}(p, u, \alpha)\right|_{u=0}
\end{aligned}
$$

using

$$
\int_{0}^{\infty} d \alpha_{1} d \alpha_{2} f\left(\alpha_{1}+\alpha_{2}\right)=\int_{0}^{\infty} \alpha d \alpha f(\alpha)
$$

Analytic continuation and some combinatorial considerations complete the proof of Equation (15).

\section{II.4. Ward Identities, etc.}

Choosing the infinitesimal transformations of some Lie-group as variations we may derive Ward identities from the action principle, Equation (13):

$$
\delta_{a} \phi_{i}(x)=t_{i j}^{a} \phi_{j}(x) \delta \varepsilon(x)
$$

with real, antihermitean matrices $t_{i j}^{a}$. We get from Equation (13a)

$$
\begin{aligned}
\delta \mathscr{L}(x)= & \left(\delta \mathscr{L} / \delta \phi_{i}(x)\right) t_{i j}^{a} \phi_{j}(x)+\left(\delta \mathscr{L} / \delta \partial_{\mu} \phi_{i}(x)\right) t_{i j}^{a} \partial_{\mu} \phi_{j}(x) \\
& \left.-\partial_{\mu}\left(\delta \mathscr{L} / \delta \partial_{\mu} \phi_{i}(x)\right) t_{i j}^{a} \phi_{j}(x)\right) \\
= & \delta_{a} \mathscr{L}(x)-\partial_{\mu} J_{\mu}^{a}(x)
\end{aligned}
$$

with the definition

$$
J_{\mu}^{a}(x)=\left(\delta \mathscr{L} / \delta \partial_{\mu} \phi_{i}(x)\right) t_{i j}^{a} \phi_{j}(x) .
$$

Isolating the source term in $\mathscr{L}=\overline{\mathscr{L}}+\int \underline{J}(x) \phi(x) d x$ we obtain from Equation (13) the Ward identity

$$
\left\langle\left(\partial^{\mu} J_{\mu}^{a}(x)-\delta_{a} \overline{\mathscr{L}}(x)-J_{i}(x) t_{i j}^{a} \phi_{j}(x)\right) \exp i \hbar^{-1} \int \mathscr{L}_{\text {int }}(x) d x\right\rangle_{+, c}^{0}=0 .
$$

The generalization of this derivation to Lagrangians including gauge fields is straightforward ${ }^{11}$. Using the so-called Slavnov transformation introduced in [3] one may in the same manner derive Slavnov-Taylor identities for non-abelian gauge theories.

A word of caution, however, has to be said about Lagrangians resp. variations involving $\gamma_{5}$. Since $\gamma_{5}$ does not anticommute with $\gamma_{\mu}$ (see Propl.1) certain simplifications usually performed in the evaluation of $\delta_{a} \mathscr{L}$ may not be possible in $n$ dimensions. This can lead to anomalies in the process of renormalization. A typical example for that is the axial vector current in theories with fermions.

The same difficulty occurs for Ward identities in super symmetric theories which depend on the identity $\gamma_{5} \psi\left(\bar{\psi} \gamma_{5} \psi\right)+\psi(\bar{\psi} \psi)=0$ for a Majorana field. For $n=4$ this follows from Fierz symmetry, a typical 4-dimensional property derived

11 We want to recall, however, that we excluded theories with massless particles 
from the completeness relation for the $\gamma$-algebra, which cannot directly be generalized to $n \neq 4$ and, therefore, may give rise to anomalies.

A similar situation arises in the derivation of trace identities from the variation $\delta \phi(x)=\left(d_{\phi}+x_{\mu} \partial^{\mu}\right) \phi(x)$. If the dimensions $d_{\phi}$ are chosen such that $\delta \mathscr{L}_{0}(x)$ is a divergence, $\delta \mathscr{L}_{\text {int }}$ will contain (apart from mass terms) a contribution proportional to $n-4$, which again will lead to anomalies in the process of renormalization. The problem of these anomalies is discussed in Section III.3.

As a second application of the action principle let us derive the renormalization group-resp. Callan Symanzik equation in the $\phi^{4}$-theory. Our discussion follows closely Ref. [11].

We take the Lagrangian

$$
\mathscr{L}(x)=((1+b) / 2) \partial_{\mu} \phi \partial^{\mu} \phi-\left(\left(m^{2}-a\right) / 2\right) \phi^{2}-((g-c) / 4 !) \phi^{4}
$$

where $a, b$, and $c$ are chosen such that the following normalization conditions are fulfilled ( $\Gamma_{N}$ denotes the 1 PI vertex functions with $N$ arguments)

$$
\begin{aligned}
& \Gamma_{2}\left(p^{2}=m^{2}\right)=0, \\
& \Gamma_{2}\left(p^{2}=\mu^{2}\right)=i\left(\mu^{2}-m^{2}\right), \\
& \left.\Gamma_{4} \text { (s.p. }\right)=-i g, \text { s.p. }=\text { symmetry point: } p_{i} p_{j}=\left(4 \delta_{i j}-1\right) \mu^{2} / 3, i, j=1, \ldots, 4 .
\end{aligned}
$$

Applying the action principle Equation (15) we find

$$
\begin{aligned}
\partial \Gamma_{N} / \partial m^{2} & =\left(\left(\partial a / \partial m^{2}-1\right) \Delta_{1}+\left(\partial b / \partial m^{2}\right) \Delta_{2}+\left(\partial c / \partial m^{2}\right) \Delta_{3}\right) \Gamma_{N}, \\
\partial \Gamma_{N} / \partial \mu^{2} & =\left(\left(\partial a / \partial \mu^{2}\right) \Delta_{1}+\left(\partial b / \partial \mu^{2}\right) \Delta_{2}+\left(\partial c / \partial \mu^{2}\right) \Delta_{3}\right) \Gamma_{N}, \\
\partial \Gamma_{N} / \partial g & =\left((\partial a / \partial g) \Delta_{1}+(\partial b / \partial g) \Delta_{2}+(\partial c / \partial g-1) \Delta_{3}\right) \Gamma_{N},
\end{aligned}
$$

where the $\Delta_{i}$ signify the insertion of the extra vertices $\Delta_{1}=i 2^{-1} \int d x \phi^{2}(x)$, $\Delta_{2}=i 2^{-1} \int d x \partial_{\mu} \phi \partial^{u} \phi$ and $\Delta_{3}=(i / 4 !) \int d x \phi^{4}(x)$ into the graphs for $\Gamma_{N}$. Putting $P=\phi$ in Equation (13) we get in addition

$$
\left(-2\left(m^{2}-a\right) \Delta_{1}+2(1+b) \Delta_{2}-4(c-g) \Delta_{3}+N\right) \Gamma_{N}=0 .
$$

Eliminating the $\Delta_{i}$ from ( $\left.31 \mathrm{~d}\right)$ in favour of the diff. operators in $(31 \mathrm{a}-\mathrm{c})$ it takes the form

$$
\begin{aligned}
& {\left[\mu^{2} \partial / \partial \mu^{2}+\varrho m^{2} \partial / \partial m^{2}+\sigma \partial / \partial g-\tau N\right] \Gamma_{N}=0,} \\
& {\left[\mu^{2} \partial / \partial \mu^{2}+m^{2} \partial / \partial m^{2}+\beta \partial / \partial g-\gamma N\right] \Gamma_{N}=\alpha m^{2} \Delta_{1} \Gamma_{N}}
\end{aligned}
$$

with some coefficients $\alpha$ depending on $m, \mu, g$ and $\beta, \gamma, \varrho, \sigma, \tau$ depending on $m / \mu$ and $g$. Applying Equation (32) to the normalization conditions Equation (30) we get

$$
\varrho=0, \quad \sigma=4 g \tau-i \mu^{2} \partial \Gamma_{4} / \partial \mu^{2} \text { (s.p.), } \quad \tau=\mu^{2}\left(\partial \Gamma_{2} / \partial \mu^{2}\right)\left(p^{2}=\mu^{2}\right) / 2 i\left(\mu^{2}-m^{2}\right)
$$

and similar for $\alpha, \beta, \gamma$.

Equations (32) are the renormalization group resp. Callan-Symanzik equation. Note that there is no hard mass insertion like in Ref. [11] involved since $\Delta_{1}$ is soft. 


\section{Dimensional Renormalization}

\section{III.1. Definition of the Renormalized Amplitudes}

As we have emphasized in Section II, the dimensionally regularized Feynman amplitudes are meromorphic functions of the complex parameter $n$. The problem of renormalization consists in subtracting the poles of these functions at $n=4$ in a way which is compatible with additive renormalization. That is, the subtractions have to be performed in a way that they correspond to counterterms in the Lagrangian. What is to be subtracted for a graph $G$ is the singular part of the amplitude for $H$ inserted into the amplitude for the reduced graph $G / H$. We will symbolize this subtraction by the application of a subtraction operator $C_{H}$ analogous to the Taylor series subtraction operator in the BPHZ scheme. The singular part of the amplitude for $H$ is defined to be the singular part of the Laurant series expansion at $n=4$ of $\overline{\mathscr{T}}_{H}$, the amplitude resulting from $\mathscr{T}_{H}$ after performance of the subtractions corresponding to all its genuine 1PI subgraphs.

Theorem 1. The singular part of the dimensionally regularized amplitude for $H$ as defined above consists of poles of order $h_{H}$ or less and is a polynomial of degree $\omega_{H}$ in the external momenta of the graph. The singular part vanishes if $H$ is superficially convergent. The amplitudes $\mathscr{R}_{G, \varepsilon}(p, n)$, remaining after performance of the subtractions corresponding to all $1 P I$ subgraphs, are analytic at $n=4$ in any order of perturbation theory. The $\lim _{\varepsilon \rightarrow 0} \mathscr{R}_{G, \varepsilon}(p, n)$ exists in $\mathscr{S}^{\prime}\left(\mathbb{R}^{4 M^{\prime}}\right)$ and is again analytic $n=4$. This limit $\mathscr{R}_{G}(p, n)$ (or its value for $n=4$ ) is the renormalized amplitude.

Remark. Clearly the overall singularity at $n=4$ of the dimensionally regularized Feynman amplitude is not a polynomial in its external momenta. This becomes only true after combination with the counterterms corresponding to all its genuine 1PI subgraphs.

It is straightforward to show that the procedure described here differs by a finite renormalization from the BPHZ scheme. Therefore, the procedure defines a renormalization fulfilling the usual requirements of causality and unitarity [5]. As usual, the physical normalization conditions can be achieved by finite counterterms in the Lagrangian.

\section{III.2. Proof of Theorem 1}

Let us first discuss the limit $\varepsilon \rightarrow 0$. Since the renormalized amplitude (for $n \neq 4$ ) is a finite sum of the unrenormalized, regularized amplitude + counterterms, each of which has a continuous $\lim _{\varepsilon \rightarrow 0}$ in $\mathscr{S}^{\prime}\left(\mathbb{R}^{4 M^{\prime}}\right)$, there is no question that also $\lim _{\varepsilon \rightarrow 0} \mathscr{R}_{G, \varepsilon}(\mathrm{p}, \mathrm{n})$ exists in $\mathscr{S}^{\prime}\left(\mathbb{R}^{4 M^{\prime}}\right)$ for $n \neq 4$. Since $\mathscr{R}_{G, \varepsilon}(p, n)$ is analytic at $n=4$, $\lim _{\varepsilon \rightarrow 0} \mathscr{R}_{G, \varepsilon}(p, n)$ can be continued to $n=4$.

In order to prove that the subtractions actually have the required properties we have to exhibit the singularities connected with all the 1PI subgraphs of a given graph $G$ and combine them with the corresponding counterterms. As usual, the complication due to overlapping divergences is resolved be the decomposition 
of the integration region in $\alpha$-space according to families of non-overlapping subgraphs. It is, however, to be remarked that in contrast to BPH-renormalization the subtraction corresponding to a particular subgraph $H$ can be completely absorbed into the integrations over those sectors related to families containing $H$, leading to the simple representation Equation (3) below.

We use maximal forests as defined in Appendix A, since they correspond directly to counterterms in the Lagrangian. They differ from the $s$-families of Speer [9] mainly by a different definition of overlapping graphs.

According to Lemma 3 the regularized Feynman amplitude can be decomposed

$$
\mathscr{T}_{G}=\lim _{\varepsilon \rightarrow 0} \mathscr{T}_{G, \varepsilon}=\lim _{\varepsilon \rightarrow 0} \int_{0}^{\infty} d \underline{\alpha} I_{G, \varepsilon}(p, \underline{\alpha})=\lim _{\varepsilon \rightarrow 0} \sum_{(\mathscr{C}, \sigma)} \int_{\mathscr{Q}(\mathscr{G}, \sigma)} d \underline{\alpha} I_{G, \varepsilon}(\underline{p}, \underline{\alpha})
$$

where the sum extends over all labelled forests $(\mathscr{C}, \sigma)$ for $G$. Resolving the recursive definition of the subtraction operators into a forest formula, anticipating that we know the action of $C_{H}$ on $I_{G}$, the renormalized amplitude can be decomposed accordingly

$$
\begin{aligned}
\mathscr{R}_{G} & =\lim _{\varepsilon \rightarrow 0} \mathscr{R}_{G, \varepsilon}=\lim _{\varepsilon \rightarrow 0} \int_{0}^{\infty} d \underline{\alpha} \sum_{F \in \mathscr{F}} \prod_{H \in F}\left(-C_{H}\right) I_{G, \varepsilon}(\underline{p}, \underline{\alpha}) \\
& =\lim _{\varepsilon \rightarrow 0} \sum_{(\mathscr{E}, \sigma)} \int_{\mathscr{D}(\mathscr{E}, \sigma)} d \underline{\alpha} \sum_{F \in \mathscr{F}} \prod_{H \in F}\left(-C_{H}\right) I_{G, \varepsilon}(\underline{p}, \underline{\alpha})
\end{aligned}
$$

where $\mathscr{F}$ is the set of all forests of non-overlapping 1 PI subgraphs of $G$. Since $C_{H}$ will be defined such that $\int_{\mathscr{Q} \mathscr{C}, \sigma)} d \alpha \prod_{H \in F}\left(-C_{H}\right) I_{G, \varepsilon}=0$ unless $F(\mathscr{C}$, we can rewrite the renormalized amplitude

$$
\begin{aligned}
\mathscr{R}_{G, \varepsilon} & =\sum_{(\mathscr{Q}, \sigma)} \int_{\mathscr{D}(\mathscr{\mathscr { C }}, \sigma)} d \underline{\alpha} \sum_{F \subset \mathscr{C}} \prod_{H \in F}\left(-C_{H}\right) I_{G, \varepsilon}(\underline{\underline{\alpha}}, \underline{)}) \\
& =\sum_{(\mathscr{Q}, \sigma)} \int_{\mathscr{D}(\mathscr{G}, \sigma)} d \underline{\alpha} \prod_{H \in \mathscr{C}}\left(1-C_{H}\right) I_{G, \varepsilon}(\underline{p}, \underline{\alpha}) .
\end{aligned}
$$

It is easy to verify that the regularized amplitude for a $1 \mathrm{PR}$ graph is the product of the regularized amplitudes for its components and of the (momentum space) propagators for all lines connecting them. This factorization is obviously maintained for the decomposition of the renormalized amplitude as given by Equations $(2,3)$ [compare Lemma 3 (a)]. We will therefore assume for the remainder of this subsection (III.2) that $G$ is $1 \mathrm{PI}$ in order to avoid complicated notation.

For each labelled forest $(\mathscr{C}, \sigma)$ we introduce the familiar scaling variables $(t, \underline{\beta})=\left(t_{H}, H \in \mathscr{C}, \beta_{\ell}, \ell \in \mathscr{L}_{G}^{\prime}=\mathscr{L}_{G} \backslash \sigma(\mathscr{C})\right)[8]$ as well as auxiliary variables $\xi_{H}, \zeta_{H}$

$$
\alpha_{\ell}=\left\{\begin{array}{l}
\prod_{H \subset H^{\prime} \in \mathscr{C}} t_{H^{\prime}}^{2}=t_{H}^{2} \xi_{H}^{2}=\zeta_{H}^{2} \text { if } \ell=\sigma(H), \quad H \in \mathscr{C}, \\
\beta_{\ell} \zeta_{H}^{2} \text { if } \quad \ell \in \mathscr{L}_{\bar{H}}^{\prime}=\mathscr{L}_{\bar{H}} \backslash \sigma(H), \quad H \in \mathscr{C},
\end{array}\right.
$$

and define $\beta_{\ell}=1$ if $\ell \in \sigma(\mathscr{C})$ for convenience. 
The image of $\mathscr{D}(\mathscr{C}, \sigma)$ under this substitution is the set $\left\{(t, \underline{\beta}): 0 \leqq t_{G}<\infty ; 0 \leqq t_{H} \leqq 1\right.$ for $H \neq G ; 0 \leqq \beta_{\ell} \leqq 1$ for $\left.\ell \in \mathscr{L}_{G}^{\prime}\right\}$.

Using Lemma 4 we may rewrite the contribution of $\mathscr{D}(\mathscr{C}, \sigma)$ to $\mathscr{T}_{G, \varepsilon}$

$$
\begin{aligned}
\int_{\mathscr{D}(\mathscr{C}, \sigma)} d \underline{\alpha} I_{G, \varepsilon}(p, \underline{\alpha})= & \prod_{\ell \in \mathscr{L}^{\prime} G} \int_{0}^{1} d \beta_{\ell} \prod_{H \in \mathscr{C}}\left\{2 \int_{0}^{\theta_{H}}\left(d t_{H} / t_{H}\right) t_{H}^{v h_{H}-\omega_{H}} Z_{H}(-i \partial / \partial \tilde{u})\right\} \\
& \cdot g_{\varepsilon}(\tilde{q}, \underline{\tilde{u}}, \underline{t}, \underline{\beta}, v) \\
= & \prod_{H \in \mathscr{C}}\left\{\int d \mu_{H} \zeta^{\nu-\omega_{\bar{H}}} Z_{H}(-i \partial / \partial \tilde{u})\right\} g_{\varepsilon}(\underline{\tilde{q}}, \underline{\tilde{u}}, \underline{t}, \underline{\beta}, v)
\end{aligned}
$$

where $v=n-4, \omega_{\bar{H}}=\omega_{H}-\sum_{H^{\prime} \in \mathcal{M}(H)} \omega_{H^{\prime}}, Z_{H}(-i \partial / \partial \tilde{u})=\prod_{\ell \in \mathscr{L}_{\bar{H}}}\left\{\zeta_{H}^{r_{\ell}} Z_{\ell}(-i \partial / \partial u)\right\}$ and $\int d \mu_{H}=2 \int_{0}^{\theta_{\boldsymbol{H}}} d t_{H} / t_{\boldsymbol{H}} \int_{0}^{1} \prod_{\ell \in \mathscr{L}^{\prime} \bar{H}} d \beta_{\ell}$ with $\theta_{G}=\infty$ and $\theta_{H}=1$ for $H \neq G$.

The $Z_{H}$ are polynomials in $\underline{t}$ and $g_{\varepsilon}$ is $C^{\infty}$ in $(t, \underline{t})$, analytic in $v$ and exponentially decreasing as $t_{G} \rightarrow \infty$. For the definition of $\tilde{H}, \tilde{q}, \underline{\tilde{u}}$ etc. see Appendix A.

Considered as a generalized function $t_{H,+}^{\lambda}$ is a meromorphic function of $\lambda$ with simple poles at all negative integers

$$
t_{+}^{v h-\omega-1}=(-)^{\omega} \delta^{(\omega)}(t) / \omega ! v h+\text { regular at } v=0 .
$$

[We put $\delta^{(\omega)}(t) / \omega !=0$ and $(d / d t)^{\omega} / \omega !=0$ for $\omega<0$.] This will enable us to define the action of the subtraction operator $C$ on the Feynman integrand. Correspondingly we can decompose the $t_{\boldsymbol{H}}$-integral in Equation (4) into

$$
\left.\left(\omega_{H} ! v h_{H}\right)^{-1}\left(d / d t_{H}\right)^{\omega_{H}} g_{\varepsilon}\left(\ldots t_{H} \ldots\right)\right|_{t_{H}=0}+\int_{0}^{\infty}(\ldots)_{\text {reg. }} d t_{H} .
$$

Due to the structure of the integrand $I_{\varepsilon}$ the first part is a polynomial of degree $\omega_{H}$ in the external momenta of $H$. The second part is analytic at $n=4$ and may be represented by the following integral, convergent for $\left|\operatorname{Re}\left(v h_{H}\right)\right|<1$

$$
\begin{aligned}
\int_{0}^{\infty}(\ldots)_{\text {reg. }} d t_{H}= & \int_{0}^{\infty} d t_{H} t_{H}^{\nu h_{H}-\omega_{H}-1}\left[g_{\varepsilon}\left(\ldots t_{H} \ldots\right)-\sum_{k=0}^{\omega_{H}-1}\left(t_{H}^{k} / k !\right) g^{(k)}\left(\ldots t_{H}=0 \ldots\right)\right. \\
& \left.-\theta\left(1-t_{H}\right)\left(t_{H}^{\omega_{H}} / \omega_{H} !\right) g^{\left(\omega_{H}\right)}\left(\ldots t_{H}=0 \ldots\right)\right] .
\end{aligned}
$$

Next we proceed to the definition of the subtraction operators $C_{H}$ acting on the Feynman integrand $I_{G, \varepsilon}$. Let us denote by $\mathscr{A}_{G}$ the formal algebra of covariants generated by $\left\{p_{i, \mu}\right\}_{i \in \mathscr{V}_{G}}$ and $\left\{u_{\ell, \mu}\right\}_{\ell \in \mathscr{L}_{G}}$ (and by $\gamma_{\mu}$ 's etc. if required) with complex coefficients depending meromorphically on $n$.

On $A \in \mathscr{A}_{G}$ we define the operator $C$ (not yet $C_{H}$ !) by

$$
C(A)=\text { sing. part at } n=4 \text { of } \operatorname{NF}\left(\left.A\right|_{\underline{u}=0}\right) .
$$

Notice that $C$ is precisely the operator to be used in a recursive definition of the counterterms. 
As a first step versus the construction of a suitable domain for the $C_{H}$ we consider the set $\mathscr{G}_{G}$ of triplets $\hat{X}=\left(X, F_{X},(\mathscr{C}, \sigma)_{X}\right)$ with $X \subset G, F_{X} \in \hat{\mathscr{F}}_{X}^{\prime}=\{$ forests of pairwise disjoint, $1 \mathrm{PI}$, proper subgraphs of $X\},(\mathscr{C}, \sigma)_{X}$ a labelled forest for $X / F_{X}$.

Next we introduce a set $\mathscr{E}_{G}$ of functions on $\mathscr{G}_{G}$ with values in $\mathscr{A}_{G}: \mathscr{E}_{G}=$ $\left\{f: \mathscr{G}_{G} \rightarrow \mathscr{A}_{G}, f(\hat{X})\right.$ depends only on the $(\underline{p}, \underline{u})$ from $\left.X / F_{X}\right\}$. As the domain for $C_{H}$ we take the subset $\mathscr{D}_{H}=\left\{f \in \mathscr{E}_{G}\right.$ : for all $\hat{X}$ of the form $\hat{X}=\left(H, \emptyset,(\mathscr{C}, \sigma)_{H}\right) C(f(\hat{X}))$ is a polynomial in $\underline{p}\}$.

One particular such function $I_{G}$ can be affiliated with the Feynman amplitude for $G . I_{G}: \hat{X} \rightarrow I_{X / F_{X}}$, represented in the form of Equation (4) in the sector $\mathscr{D}(\mathscr{C}, \sigma)_{X}$. The meromorphic dependence of the coefficients on $n$ is achieved by interpreting the fractional powers of $t$ 's in the distributional sense as displayed in Equations $(5,6)$.

On $\mathscr{D}_{H}$ the operator $C_{H}$ is defined by

$$
\left(C_{H} f\right)(\hat{X})=\left\{\begin{array}{l}
f(\hat{X}) \text { if either } X \cap H=\emptyset \text { or } H \varsubsetneqq H^{\prime} \text { for some } H^{\prime} \in F_{X} \\
C(f(\hat{X})) \text { if } X=H \text { and } F_{X}=\emptyset \\
U_{H}\left(C\left(f\left(\hat{H}_{0}\right)\right)\right) f(\widehat{X: H}) \text { if } H \notin F_{X}, F_{X} \cup\{H\} \in \mathscr{F}_{X}^{\prime} \\
0 \text { else }
\end{array}\right.
$$

where $\hat{H}_{0}=\left(H, \emptyset,(\mathscr{C}, \sigma)_{H}\right)$ and $\widehat{X: H}=\left(X, F_{X} \cup\{H\},(\mathscr{C}, \sigma)_{X: H}\right)$ with $(\mathscr{C}, \sigma)_{H},(\mathscr{C}, \sigma)_{X: H}$ the pair of labelled forests for $H$ resp. $X / F_{X} \cup\{H\}$ corresponding to $(\mathscr{C}, \sigma)_{X}$ (see Appendix A). $U_{H}$ is defined in Appendix B.

Equation (3) is to be interpreted as $\left.\sum_{(\mathscr{C}, \sigma)} \int d \underline{t} d \underline{\beta}\left[\prod_{H \in \mathscr{C}}\left(1-C_{H}\right) I_{G}\right](G, \emptyset,(\mathscr{C}, \sigma))\right|_{\underline{u}=0}$ where the factors $\left(1-C_{H}\right)$ are to be ordered such that those for smaller graphs act first.

The main problem in combining the singular part of the integral with the expression for the counterterm for $H$ inserted into $G / H$ comes from the fact that the coefficient of the pole at $v=0$ exhibited in Equation (4) depends on the number of loops of $H$. The subtractions corresponding to genuine subgraphs of $H$ can be interpreted as counterterms inserted into reduced graphs and these reduced graphs will have fewer loops than $H$ has. Therefore the term $t^{v h}$ in Equation (4) will be replaced by a sum of terms with different exponents and furthermore with coefficients which have poles at $v=0$. The following definition will be useful to trace such sums through the recursion.

Given a maximal forest $\mathscr{C}$ for $G$, we define sets of functions $J_{H}^{K}$ (for $0 \leqq K<h_{H}$ ) and $\tilde{J}_{H}^{K}\left(\right.$ for $\left.0 \leqq K \leqq h_{H}\right)$ for all $H \in \mathscr{C}$

$$
\begin{aligned}
& J_{H}^{K}=\left\{f(\xi, v): f(\xi, v)=\xi^{v} \prod_{H^{\prime} \in \mathscr{M}(H)} g_{H^{\prime}}(\xi, v) \text { with } g_{H^{\prime}} \in \tilde{J}_{H}^{K_{H^{\prime}}}, K=\sum_{H^{\prime} \in \mathcal{M}(H)} K_{H^{\prime}}\right\} ; \\
& \tilde{J}_{H}^{K}=\left\{g(\xi, v): g \text { or } \xi^{v} g \text { with } g \in J_{H}^{K} \text { or } g(\xi, v)=\int_{1}^{\xi} d x / x f(x, v) \text { with } f \in J_{H}^{K-1}\right\} .
\end{aligned}
$$

If $h_{H}=1$ (i.e. $\mathscr{M}(H)=\emptyset$ ) there is only $K=0$ and $J_{H}^{0}=\left\{\xi^{\nu}\right\}$. 
Lemma 2. Let $f(\xi, v)$ be any element of $J_{H}^{K}$, then
(a) $f(\xi, v)=v^{-K} \sum_{m=1}^{h_{H}} c_{m} \xi^{v m} \quad$ with some constants $c_{m}$;
(b) $f(\xi, 0)=c(\ln \xi)^{K} \quad$ with some constant $c$;
(c) $f(\xi t, v)=\sum_{j} f_{1 j}(\xi, v) f_{2 j}(t, v)$

with $f_{i j} \in J_{H}^{K_{i j}}$ such that $K_{1 j}+K_{2 j}=K$;

(d) $J_{H}^{0}=\left\{\xi^{m v}, m=1, \ldots, h_{H}\right\}$.

Proof. All statements are true if $h_{H}=1$. (a), (b) and (d) follow easily from the resursive definition of the sets $J_{H}^{K}$. Assume that (c) holds for all $H^{\prime}$ which are genuine subgraphs of $H$ and let $h(x, v) \in J_{H^{\prime}}^{K-1}$. Then

$$
\begin{aligned}
\int_{1}^{\xi t}(d x / x) h(x, v) & =\int_{1}^{t}(d x / x) h(x, v)+\int_{1}^{\xi}(d x / x) h(x t, v) \\
& =\int_{1}^{t}(d x / x) h(x, v)+\sum_{j} \int_{1}^{\xi}(d x / x) h_{1 j}(x, v) h_{2 j}(t, v)
\end{aligned}
$$

with $h_{i j} \in J_{H^{\prime}}^{K_{i j}}$ such that $K_{1 j}+K_{2 j}=K-1$. Insertion of this decomposition into the definition of $J_{H}^{K}$ proves the statement (c) for $H$.

It should be noted that the pole of order $K$ at $v=0$, indicated by the decomposition (7) is not really there. This is made explicit by Equation (8).

Now we are ready to prove

Proposition 3. Consider any $\mathscr{X}_{0} \in \hat{\mathscr{F}}_{G}^{\prime}, \mathscr{X}_{0} \subset \mathscr{C}$ and define

$$
\mathscr{X}=\left\{H^{\prime} \in \mathscr{C}: H^{\prime} \subset H \text { for some } H \in \mathscr{X}_{0}\right\} \text {. }
$$

After performance of all subtractions corresponding to subgraphs $H \in \mathscr{X}$, the contribution of $(\mathscr{C}, \sigma)$ to $\mathscr{R}_{\mathbf{G}, \varepsilon}$ is a sum of terms of the form

$$
\begin{aligned}
& \prod_{H \in \mathscr{C} \backslash \mathscr{X}}\left\{\int d \mu_{H}\left(1-C_{H}\right) \zeta_{H}^{\nu-\omega_{\bar{H}}} Z_{H}(-i \partial / \partial \tilde{u})\right\} \prod_{H \in \mathscr{X}_{0}}\left\{\xi_{H}^{-\omega_{H}} g_{H}\left(\xi_{H}, v\right)\right\} \\
& \left.\cdot g_{\mathscr{X}}(\tilde{\underline{q}}, \underline{\tilde{u}}, \underline{t}, \underline{\beta}, v)\right|_{\tilde{\underline{u}}=0}
\end{aligned}
$$

where $(t, \underline{\beta})$ and $\underline{\tilde{u}}$ are scaling variables and scaled $u$ 's for $G / \mathscr{X}_{0}$ (i.e. $\underline{u}_{H}$ for $H \in \mathscr{X}$ are already set to zero) and $\tilde{q}$ are scaled momenta appropriate for the family $\mathscr{C} \backslash \mathscr{X}$. $g_{H} \in \tilde{J}_{H}^{K}$ for some $K$ and $g_{\mathscr{X}}$ is some element of the abstract algebra of covariants with complex coefficients which are $C^{\infty}$ in $(\underline{t}, \underline{\beta})$, analytic at $v=0$ and, due to $\varepsilon>0$, exponentially decreasing as $t_{G} \rightarrow \infty$.

Proof (by induction with respect to $\sum_{H \in \mathscr{X}_{0}} h_{H}=|\mathscr{X}|$ ).

i) The statement certainly holds for $\mathscr{X}=\emptyset$ [compare Eq. (4)].

ii) Consider some $\mathscr{X} \subset \mathscr{C}(\mathscr{X} \neq \varnothing) \mathscr{X}_{0} \in \hat{\mathscr{F}}_{G}$, choose any $H \in \mathscr{X}_{0}$ and assume that the statement is true for $\mathscr{X}_{0}^{\prime}=\mathscr{M}(H) \cup \mathscr{X}_{0} \backslash\{H\}$ (i.e. $\mathscr{X}^{\prime}=\mathscr{X} \backslash\{H\}$ ). If $\mathscr{X}_{0}=\{G\}$ we will prove Theorem 1, otherwise we will show that the proposition holds for $\mathscr{X}_{0}$ as well. 
The relevant part of Equation (11) is

$$
\left.\int d \mu_{H}\left(1-C_{H}\right) \zeta_{H}^{\nu-\omega_{\bar{H}}} Z_{H}(-i \partial / \partial \tilde{u}) \prod_{\boldsymbol{H}^{\prime} \in \mathscr{M}(\boldsymbol{H})}\left(\xi_{H^{\prime}}^{-\omega_{H^{\prime}}} g_{H^{\prime}}\left(\xi_{H^{\prime}}, v\right)\right) g_{X^{\prime}}\right|_{\underline{\underline{u}}_{H}=0}
$$

First we notice that $\xi_{H^{\prime}}=\zeta_{H}=t_{H} \xi_{H}$ for $H^{\prime} \in \mathscr{M}(H)$. Evaluating the derivatives with respect to $\underline{\tilde{u}}_{H}$, setting $\underline{\tilde{u}}_{H}=0$ and integrating over $\beta$ 's we obtain

$$
(12)=\left(1-C_{H}\right) \int_{0}^{\infty}\left(d t_{H} / t_{H}\right)\left(\xi_{H} t_{H}\right)^{-\omega_{H}} f\left(\xi_{H} t_{H}, v\right) g^{\prime}\left(t_{H}, \underline{\tilde{q}}, \underline{\tilde{u}}, \underline{\underline{\beta}}, \underline{\beta}, v\right)
$$

where $f \in J_{H}^{K}$ and we have replaced the variables $\left(\underline{\tilde{q}}^{\prime}, \underline{\tilde{u}}^{\prime}, \underline{t}^{\prime}, \underline{\beta}^{\prime}\right)$, appropriate for $\mathscr{X}^{\prime}$, by those for $\mathscr{X}$. In particular $\underline{q}_{H}=\left(\tilde{q}_{H}^{\prime} / t_{H}, \tilde{q}_{H^{\prime}}^{\prime}, H^{\prime} \in \mathscr{M}(H)\right)$ and therefore $g^{\prime}$ is again $C^{\infty}$ in $\left(t_{H}, \underline{t}, \underline{\beta}\right)$ and analytic at $v=0$. Furthermore $g^{\prime}=0$ for $t_{H}>1$ unless $H=G$. Using Equations $(5,7)$ we get

$$
\begin{aligned}
(12)= & \left(1-C_{H}\right) \xi_{H}^{-\omega_{H}}\left\{v^{-K-1} \sum_{m=0}^{h_{H}} c_{m} m^{-1} \xi_{H}^{v m}\left[\frac{1}{\omega_{H} !}\left(d / d t_{H}\right)^{\omega_{H}} g^{\prime}\right] t_{H}=0\right. \\
& \left.+\int_{0}^{\infty}\left(\left(d t_{H} / t_{H}\right) t_{H}^{-\omega_{H}} f\left(\xi_{H} t_{H}, v\right) g^{\prime}\right)_{\text {reg. }}\right\} .
\end{aligned}
$$

In contrast to Equation (7) there is now really a pole of order $K+1 \leqq h_{H}$ at $\nu=0$. If $H=G$ then $\xi_{H}=1, \tilde{q}=\underline{q}$ and there are no $\underline{\tilde{u}}$ 's left in $g^{\prime}$. As shown in Appendix B

$$
P(q)=\left[\left(\omega_{H} !\right)^{-1}\left(d / d t_{H}\right)^{\omega_{H}} g^{\prime}\right]_{t_{H}=0}
$$

is a polynomial of degree $\omega_{H}$ in the external momenta $q$ (a homogeneous polynomial in momenta, masses and $\sqrt{\varepsilon}$ if all $Z_{\ell}$ are homogeneous in these variables). We reduce $P(q)$ to NF in order to make its $v$-dependence explicit. We do, however, not interprete $P(q)$ as a 4-dimensional covariant at this point. Due to the exponential decrease of $g^{\prime}$ the regularized integral is absolutely convergent and analytic in $v$. Using the Taylor operator $T_{v}^{K}$ of degree $K$ in $v$ we obtain

$$
v^{-K-1} \sum_{m} c_{m} m^{-1} T_{v}^{K} P(\underline{q})
$$

as one term in the contribution of $(\mathscr{C}, \sigma)$ to the counterterm for $H$ which is annihilated by $\left(1-C_{H}\right)$. The contribution to $\mathscr{R}_{H, \varepsilon}$

$$
(12)=\sum_{m} c_{m} m^{-1} v^{-K-1}\left(1-T_{v}^{K}\right) P(q)+\int_{0}^{\infty}\left(\left(d t_{H} / t_{H}\right) t_{H}^{-\omega_{H}} f\left(t_{H}, v\right) g^{\prime}\right)_{\text {reg. }}
$$

is analytic at $v=0(n=4)$ by construction. This proves Theorem 1 , once we have completed the proof of Proposition 3.

If $H \neq G$ we use Lemma 5 to rewrite $\left[\left(\omega_{H} !\right)^{-1}\left(d / d t_{H}\right)^{\omega_{H}} g^{\prime}\right]_{t_{H}=0}$ as the amplitude for $G / H$ with the polynomial $P$ [Eq. (15)] inserted as vertex part:

$$
\left[\left(\omega_{H} !\right)^{-1}\left(d / d t_{H}\right)^{\omega_{H}} g^{\prime}\right]_{t_{H}=0}=\tilde{P}\left(\tilde{q}_{H},-i \partial / \partial \underline{\tilde{u}}_{G / H}\right) g_{G / H}
$$

where

$$
\tilde{P}\left(\underline{\tilde{q}}_{H},-i \partial / \partial \underline{\tilde{u}}_{G / H}\right)=\xi_{H}^{\omega_{H}} P\left(q_{H}-i e_{G H}^{+} \partial / \partial u_{G}^{+}\right)
$$

is a polynomial in $\underline{\tilde{q}}, \partial / \partial \underline{\tilde{u}}$ and $\underline{t}$. 
Let $\left(\left(\mathscr{C}_{1}, \sigma_{1}\right),\left(\mathscr{C}_{2}, \sigma_{2}\right)\right)$ be the pair of labelled forests for $G / H$ and $H$ corresponding to $(\mathscr{C}, \sigma)$. Due to Lemma $5 g_{G / H}$ is the precise analogue of $g_{\mathscr{X}}$ in Equation (11) but for the contribution of $\left(\mathscr{C}_{1}, \sigma_{1}\right)$ to $\mathscr{R}_{G / H, \varepsilon}$ after subtracting all counterterms corresponding to subgraphs contained in $\mathscr{X} \cap \mathscr{C}_{1}$. The counterterm for $H$ can now be inserted into the partially subtracted amplitude for $G / H$, i.e. $\left(1-C_{H}\right)$ can be evaluated and we obtain

$$
\text { (12) }=\xi_{H}^{-\omega_{H}}\left\{v^{-K-1} \sum_{m} c_{m} m^{-1}\left[\left(\xi_{H}^{v m}-T_{v}^{K}\right) \tilde{P}\right] g_{G / H}+\int_{0}^{\infty}(\ldots)_{\text {reg. }}\right\} \text {. }
$$

Using Equation (7) we may rewrite the first part as

$$
\begin{aligned}
v^{-K-1} & \sum_{m} c_{m} m^{-1}\left[\left(\xi_{H}^{v m}-T_{v}^{K}\right) \tilde{P}\right] g_{G / H} \\
= & v^{-K-1} \sum_{m} c_{m} m^{-1}\left\{\left[\left(\xi_{H}^{v m}-1\right) T_{v}^{K}+\xi_{H}^{v m}\left(1-T_{v}^{K}\right)\right] \tilde{P}\right\} g_{G / H} \\
= & \int_{1}^{\xi_{H}}(d t / t) f(t, v)\left(T_{v}^{K} \tilde{P}\right) g_{G / H}+\sum_{m} c_{m} m^{-1} \xi_{H}^{v m}\left[v^{-K-1}\left(1-T_{v}^{K}\right) \tilde{P}\right] g_{G / H}
\end{aligned}
$$

and evaluate the derivatives with respect to $\underline{\tilde{u}}$ contained in $\tilde{P}$. For the regularized integral we use Equation (9) to get

$$
\begin{aligned}
\int_{0}^{\infty}(\ldots)_{\text {reg. }}= & \sum_{j} f_{1 j}\left(\xi_{H}, v\right)\left\{\int_{0}^{1}(d t / t) f_{2 j}(t, v) t^{-\omega_{H}}\left(1-T_{t}^{\omega_{H}}\right) g^{\prime}\right. \\
& \left.-\int_{1}^{\infty}(d t / t) f_{2 j}(t, v) t^{-\omega_{H}}\left(T_{t}^{\omega_{H}-1} g^{\prime}\right)\right\}
\end{aligned}
$$

where both integrals are absolutely convergent and yield a function $C^{\infty}$ in $(t, \underline{\beta})$ and analytic at $v=0$. Collecting all contributions of Equations $(18,19)$ we arrive at

$$
(12)=\xi_{H}^{-\omega_{H}} \sum g_{H}\left(\xi_{H}, v\right) g_{X}(\underline{\tilde{q}}, \underline{\tilde{u}}, \underline{\underline{\beta}}, \underline{\beta}, v)
$$

where each $g_{H}$ and $g_{\mathscr{X}}$ has all the properties required in Equation (11). Finally combining Equation (20) with all those terms not contained in Equation (12) we are led back to an expression of the form (11) but now for the set $\mathscr{X}_{0}=\{H\} \cup$ $\mathscr{X}_{0}^{\prime} \backslash \mathscr{M}(H)$. This completes the proof of both Proposition 3 and Theorem 1.

As a final remark it should be added that whenever we use the word counterterm it refers to the ones which are singular for $n=4$ and not to the additional finite counterterms which occur explicitly in the Lagrangian and serve to fix the physical normalization conditions.

\section{III.3. The Renormalized Action Principle}

Having defined the renormalized amplitudes in a satisfactory way we have to ask about the validity of the action principle in the renormalized theory, which is answered by 
Theorem 2. The action principle, Equations (II.13-15), remains valid without any change for the renormalized theory (for $n \neq 4$ ), if there is no explicit $n$-dependence involved. In particular, the classical equations of motion remain valid without any modification.

The reservation made about an explicit $n$-dependence is to be understood in the following sense. The Action Principle leads to certain linear relations between regularized Feynman amplitudes reduced to their resp. NF. If these relations have $n$-independent coefficients they remain true for the renormalized amplitudes. In contrast, $n$-dependent coefficients are generally the source of anomalies, i.e. terms absent in the tree approximation. Such $n$-dependence can arise in two ways. Either the variation $\delta \phi$ depends explicitly on $n$, as for dilatations (the dimension $d_{\phi}$ of the field $\phi$ depends on $n$ ) or the $n$-dependence comes in during the reduction to NF in the course of eliminating $\hat{0}$-type covariants. A typical case for the latter situation is the Adler anomaly. $\left\{\gamma_{\mu}, \gamma_{5}\right\}=2 \hat{\gamma}_{\mu} \gamma_{5}$ can lead to terms containing $\hat{g}_{\mu \mu}=n-4$. More generally one can say that operators like $\left(\bar{\psi}\left\{\gamma_{\mu}, \gamma_{5}\right\} \psi\right)^{\text {ren. }}$ or $\left((n-4) \phi^{4}\right)^{\text {ren. }}$ vanishing in the tree approximation are non-zero due to radiative corrections.

As was shown in Section II.3 the proof of the action principle is based essentially on the equality of two regularized amplitudes, one for a graph $G$ with a factor $Z_{\ell}=i\left(\square+m^{2}\right)$ and the other one for a graph $G / \ell$ where the line $\ell$ is contracted to a point. Assume this equality still holds for $\overline{\mathscr{T}}_{G}$, i.e. after performing all subtractions for genuine subgraphs, then uniqueness of the Laurant expansion guarantees that also the finite parts (i.e. the renormalized amplitudes) are equal and, therefore, satisfy the action principle. Here we have to use the convention that any parameter of the Lagrangian which might depend implicitly on $n$ is not subject to the Laurant expansion.

If the maximal forests for the two graphs $G$ and $G / \ell$ are in one to one correspondence we can immediately justify our assumption. Either a particular subgraph $H$ does not contain the line $\ell$ : in this case the same singular part of the amplitude for $H$ has to be inserted into the amplitudes for two reduced graphs which are equal. Or the line $\ell$ is contained in the subgraph $H$ of $G$ : then the subtractions corresponding to $H$ and $H / \ell$, being the singular part of two equal amplitudes, are equal and are inserted into the amplitude for the same reduced graph $G / H$. This again justifies recursively the above assumption, provided the maximal forests or equivalently the forests of non-overlapping 1PI subgraphs for $G$ and $G / \ell$ are in one to one correspondence.

There will, however, in general be no such one to one correspondence for the following two reasons:

i) There may exist 1PI subgraphs $H$ of $G$ which contain both vertices of $\ell$ but not the line $\ell$ itself. They do not have any corresponding subgraph in $G / \ell$.

ii) There may be 1 PR subgraphs $H$ of $G$ such that $H / \ell$ becomes 1 PI in $G / \ell$.

Figures 1 and 2 exemplify these two possibilities. All other 1PI subgraphs of $G$ are in one to one correspondence with 1 PI subgraphs of $G / \ell$.

The first case is very easily resolved for dimensional renormalization; subtractions for subgraphs like $H$ in Figure 1 may just be omitted since they do not contribute to this particular amplitude. They do, however, contribute if there is 


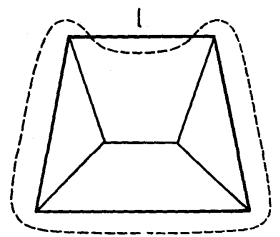

$\mathrm{H \subset G}$

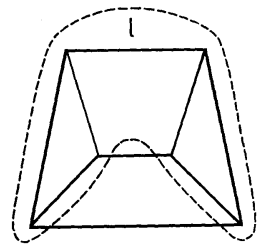

a) $H \subset G$

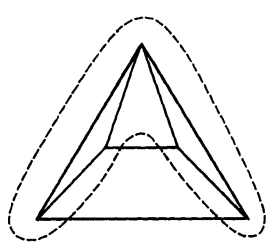

b) $H \subset G / I$
Fig. 1. Example of a subgraph $H \subset G$, which is 1PI, contains both endpoints of line $\ell$, but not line $\ell$

Fig. 2. Example of a subgraph $H \subset G$, which is $1 \mathrm{PR}$ in $G(\mathbf{a})$, but becomes $1 \mathrm{PI}$ in $G / \ell$ (b)

no factor $\left(\square+m^{2}-i \varepsilon\right)$ for line $\ell$. This is the reason why we do not use any normal ordering at all in the Gell-Mann-Low expansion (II.12). Since $\ell$ is a loop line in $G / H$, the contribution of $C_{H}$ to $\mathscr{R}_{G}$ would be proportional to

$$
\begin{gathered}
\left.\int_{0}^{\infty} d \alpha \alpha^{-n / 2} Z^{\prime}(\partial / \partial u)\left(\square_{u}+m^{2}-i \varepsilon\right) \exp \left[-i\left(\alpha\left(m^{2}-i \varepsilon\right)+u^{2} / 4 \alpha\right)\right]\right|_{u=0} \\
=\left.i \int_{0}^{\infty} d \alpha(\partial / \partial \alpha) \alpha^{-n / 2} Z^{\prime}(\partial / \partial u) \exp \left[-i\left(\alpha\left(m^{2}-i \varepsilon\right)+u^{2} / 4 \alpha\right)\right]\right|_{u=0} .
\end{gathered}
$$

Assuming that $Z^{\prime}$ is a polynomial of degree $2 k$ or less and performing the differentiations with respect to $u$, this gives

$$
\begin{aligned}
& \int_{0}^{\infty} d \alpha(\partial / \partial \alpha) \alpha^{-n / 2-k} Z^{\prime \prime}(\alpha) \exp \left[-i \alpha\left(m^{2}-i \varepsilon\right)\right] \\
& =\left.\alpha^{-n / 2-k} Z^{\prime \prime}(\alpha) \exp \left[-i \alpha\left(m^{2}-i \varepsilon\right)\right]\right|_{\alpha=0} ^{\alpha=\infty}=0 .
\end{aligned}
$$

Here we have used that the integral is defined by analytic continuation in $n$ and vanishes identical for $\operatorname{Re}(n)<-2 k$. The analogous problem has been discussed by Lam [8] for Zimmermann's renormalization scheme (normal product algorithm) $[14,15]$ whereas the problem of subtractions, related to subgraphs like the ones shown in Figure 2, has not been discussed in this context. It is shown in Appendix $C$ that the classical equations of motion are valid also for this case without any modification. This is due to a special property of our subtraction operator $C_{H}$. Assume a graph $H=H_{1} \cup H_{2}$ such that $H_{1}$ and $H_{2}$ overlap at a single vertex. The Feynman integrand for $H$ factorizes $I_{H}=I_{H_{1}} \cdot I_{H_{2}}$ and the subtraction operators satisfy

$$
\left(-C_{H}\right)\left(1-C_{H_{1}}-C_{H_{2}}\right)=\left(-C_{H_{1}}\right)\left(-C_{H_{2}}\right) \text {. }
$$

This is slightly stronger than the unitarity requirement which allows an additional finite counterterm. The proof in Appendix $\mathrm{C}$ shows, again in a recursive 
way, that the whole sum (3) for $\mathscr{R}_{G / \ell}$ may be rewritten such that we finally get the analogous sum but over all forests of $G$. Therefore, the classical equations of motion are valid for the renormalized (amplitudes of) composite operators.

Acknowledgements. We are indebted to K. Symanzik for pointing out the relevance of Equation (II.32 b) to us. Considerable improvements in the formulation of Section III and Appendix B are due to many discussions with E. R. Speer

\section{Appendix A. Feynman Graphs}

In this Appendix we have collected all relevant definitions and properties concerning Feynman graphs, following essentially the notation of Refs. [9] and [17].

A Feynman graph $G$ is a collection of vertices $\left\{V_{1}, \ldots, V_{M}\right\}=\mathscr{V}_{G}$ and of lines $\left\{\ell_{1}, \ldots, \ell_{L}\right\}=\mathscr{L}_{G}$ together with a rule assigning to each line $\ell$ an initial vertex $V_{i}$ and a final vertex $V_{f_{\ell}}$. The corresponding incidence matrix $e_{G}$ is defined by

$$
e_{\ell i}=\left\{\begin{aligned}
1 & \text { if } V_{i}=V_{f_{e}} \neq V_{i_{e}} \\
-1 & \text { if } V_{i}=V_{i_{\iota}} \neq V_{f_{c}} \\
0 & \text { otherwise }
\end{aligned}\right.
$$

A subgraph $G^{\prime} \subset G$ is a subset of lines and vertices of $G$ which build again a Feynman graph in the above sense. A generalized vertex $G^{\prime}$ of $G$ is a subgraph of $G$ containing with any pair of vertices all lines in $G$ joining them.

A Feynman graph $G$ is called connected if rank $(e)=M-1$, irreducible if it stays connected by removal of any one line or vertex, one-particle irreducible (1PI) if it is connected and stays so after removal of any one line, one-particle reducible (1PR) if it is not $1 \mathrm{PI}$, non-empty if $\mathscr{V}_{G} \neq \emptyset$, non-trivial if $\mathscr{L}_{G} \neq \emptyset$. The maximal non-empty connected subgraphs of $G$ are called its connectivity components (c-components).

Two subgraphs $G_{1}, G_{2} \subset G$ are called non-overlapping $\left(G_{1} \otimes G_{2}\right)$ if either $G_{1} \subset G_{2}$, $G_{2} \subset G_{1}$ or $G_{1} \cap G_{2}=\emptyset$, otherwise they are overlapping $\left(G_{1} \circ G_{2}\right)$.

For any $G^{\prime} \subset G$ the reduced graph $G / G^{\prime}$ is the graph obtained from $G$ by removal of all lines of $G^{\prime}$ and replacement of all the vertices of each $c$-component of $G^{\prime}$ by one new vertex. For any set $\mathscr{M}$ of pairwise disjoint subgraphs of $G, G / \mathscr{M}$ is defined as the obvious extension of this definition.

In what follows $G$ is a connected Feynman graph. The number $h_{G}=L-M+1$ is called the connectivity (or number of independent loops). A tree $T$ in $G$ is a maximal subgraph of $G$ with $h_{T}=0$. A $K$-tree $(K \geqq 2)$ is a tree minus $K-1$ lines. We call the maximal non-trivial 1 PI subgraphs of $G$ the (1PI-) components of $G$.

Let $\left\{\alpha_{\ell}\right\}$ be a set of $L$ variables then we define a $L \times L$ matrix $\alpha$ by $\alpha_{\ell \ell^{\prime}}=\alpha_{\ell} \delta_{\ell \ell^{\prime}}$ and a $M \times M$ matrix $A$ by

$$
A_{i j}=\left(e^{+} \alpha^{-1} e\right)_{i j}=\sum_{\ell=1}^{L} e_{\ell i} e_{\ell j} \alpha_{\ell}^{-1} .
$$

The incidence matrix for a connected graph has rank $M-1$, satisfying $\sum_{i=1}^{M} e_{\ell i}=0$, i.e. the vector $n=\left(\begin{array}{c}1 \\ \vdots \\ 1\end{array}\right)$ is mapped to zero by $e$. Correspondingly $A$ is singular 
since $A n=0$. Let us denote by $E=\left\{(p): \sum_{i=1}^{M} p_{i}=0\right\}$ the hyperplane orthogonal to $n$ and by $e_{E}$ resp. $A_{E}$ the restriction of $e$ resp. $A$ to $E$. Then $A_{E}$ is non-singular and we find $A_{E}=e_{E}^{+} \alpha^{-1} e_{E}$. If we parametrize $E$ by $\left\{p_{1}, \ldots, \hat{p}_{k}, \ldots, p_{M}\right\}$-where $\hat{p}_{k}$ means that $p_{k}$ is missing-we find

$$
\left(A_{E}^{-1}\right)_{i j}=A\left(\begin{array}{c}
k i \\
k j
\end{array}\right) / A\left(\begin{array}{l}
k \\
k
\end{array}\right) \quad \text { where } \quad A\left(\begin{array}{l}
i_{1}, \ldots, i_{r} \\
j_{1}, \ldots, j_{r}
\end{array}\right) \text { is the corresponding }
$$

minor of $A$.

The so-called Symanzik functions of $G, d(\alpha)=A\left(\begin{array}{l}k \\ k\end{array}\right) \prod_{\ell \in \mathscr{L}_{G}} \alpha_{\ell}$ and $D_{i j}^{k}(\alpha)=A\left(\begin{array}{c}k i \\ k j\end{array}\right)$ - $\prod_{\ell \in \mathscr{L}_{G}} \alpha_{\ell}$, can be represented in the form $d(\alpha)=\sum_{T} \prod_{\ell \notin T} \alpha_{\ell}$ where the sum runs over all trees $T$ in $G$, and $D_{i j}^{k}(\alpha)=\sum_{T_{2}} \prod_{\ell \notin T_{2}} \alpha_{\ell}$ where the sum is running over all 2-trees $T_{2}$ in $G$ which separate $V_{k}$ from $V_{i}$ and $V_{j}$. Note that $d(\alpha)$ and the quadratic form $p^{+} A_{E}^{-1} p=\sum_{i, j \neq k}\left(D_{i j}^{k}(\alpha) / d(\alpha)\right) p_{i} p_{j}$ are independent of $k$ (for $p \in E$ ).

Let $G$ be a connected graph with components $G_{1}, \ldots, G_{n}$. A maximal forest for $G$ is a maximal set of non-trivial non-overlapping 1 PI subgraphs of $G$. For any maximal forest $\mathscr{C}$ and any $H \in \mathscr{C}$ let $\mathscr{M}(H)$ be the set of all maximal elements of $\mathscr{C}$ properly contained in $H$ and let $\bar{H}$ be $H / \mathscr{M}(H)$. A labelled forest is a pair $(\mathscr{C}, \sigma)$ consisting of a maximal forest $\mathscr{C}$ and a mapping $\sigma: \mathscr{C} \rightarrow \mathscr{L}_{G}$ such that $\sigma(H) \in \mathscr{L}_{\bar{H}}$ for all $H \in \mathscr{C}$. Note that $\mathscr{M}(H)$ is uniquely determined by $H$ and $\sigma(H)$.

For any labelled forest $(\mathscr{C}, \sigma)$ we define $\mathscr{D}(\mathscr{C}, \sigma)$ to be the subset of $\alpha$-space given by

$$
\mathscr{D}(\mathscr{C}, \sigma)=\left\{\left(\alpha_{1}, \ldots, \alpha_{L}\right): \alpha_{\ell} \geqq 0 \text { for all } \ell ; \quad \alpha_{\ell} \leqq \alpha_{\sigma(H)} \text { for } \ell \in H \in \mathscr{C}\right\} .
$$

Lemma 3. (a) Any maximal forest $\mathscr{C}$ for $G$ is a disjoint union $\mathscr{C}=\bigoplus_{i=1}^{n} \mathscr{C}_{i}$ of maximal forests $\mathscr{C}_{i}$ for the components $G_{i}$ of $G ; G_{i} \in \mathscr{C}_{i}$;

(b) any maximal forest $\mathscr{C}$ for $G$ can be labelled;

(c) for any $1 P I$ subgraph $H$ of $G$ there is a natural one to one correspondence between labelled forests $(\mathscr{C}, \sigma)$ for $G$ such that $H \in \mathscr{C}$ and pairs $\left(\left(\mathscr{C}_{1}, \sigma_{1}\right),\left(\mathscr{C}_{2}, \sigma_{2}\right)\right)$ of labelled forests for $\mathrm{G} / \mathrm{H}$ and $\mathrm{H}$;

(d) any maximal forest for $G$ has exactly $h_{G}$ elements;

(e) $G-\sigma(\mathscr{C})$ is a tree in $G$;

(f) $\bigcup_{(\mathscr{C}, \sigma)} \mathscr{D}(\mathscr{C}, \sigma)=\left\{\left(\alpha_{\ell}\right): \alpha_{\ell} \geqq 0\right.$ for all $\left.\ell\right\}$;

(g) for $(\mathscr{C}, \sigma) \neq\left(\mathscr{C}^{\prime}, \sigma^{\prime}\right), \mathscr{D}(\mathscr{C}, \sigma) \cap \mathscr{D}\left(\mathscr{C}^{\prime}, \sigma^{\prime}\right)$ is a set of Lebesgue measure zero (as subset of $\mathbb{R}^{L}$ ).

Proof. (a)Follows directly from the definition.

(b) Let $H \in \mathscr{C}$ and $\mathscr{M}(H)=\left\{H_{1}, \ldots, H_{m}\right\}$. We first observe that $\bar{H}$ is non-trivial. For $m \leqq 1$ this follows directly from the definition of $\mathscr{M}(H)$. For $m \geqq 2$ the $H_{i}$ are pairwise disjoint (no common vertices and no common lines), therefore $H$ contains lines which are in no one $H_{i}$. Choose any line of $\bar{H}$ as $\sigma(H)$. 
(c) For any $(\mathscr{C}, \sigma)$ such that $H \in \mathscr{C}$ chose

$$
\begin{gathered}
\mathscr{C}_{1}=\left\{H^{\prime}: H^{\prime} \in \mathscr{C} \text { and } H^{\prime} \cap H=\emptyset ; \sigma_{1}\left(H^{\prime}\right)=\sigma\left(H^{\prime}\right)\right. \text { or } \\
\left.H^{\prime}=H^{\prime \prime} / H, H \underset{\mp}{\subset} H^{\prime \prime} \in \mathscr{C} ; \sigma_{1}\left(H^{\prime}\right)=\sigma\left(H^{\prime \prime}\right)\right\} ; \\
\mathscr{C}_{2}=\left\{H^{\prime} \in \mathscr{C}: H^{\prime} \subset H ; \sigma_{2}\left(H^{\prime}\right)=\sigma\left(H^{\prime}\right)\right\} .
\end{gathered}
$$

One easily verifies the one to one correspondence and, furthermore, if $(\mathscr{C}, \sigma)$ is a labelled forest so are $\left(\mathscr{C}_{1}, \sigma_{1}\right),\left(\mathscr{C}_{2}, \sigma_{2}\right)$ and vice versa.

(d) Due to (a) it is sufficient to prove the statement for $G 1 \mathrm{PI}$. It trivially holds for $h_{G}<2$. Let $h_{G}=n \geqq 2$ and assume the statement holds for all 1PI graphs $G^{\prime}$ with $h_{G^{\prime}}<n$. Any $\mathscr{C}$ for $G$ contains at least one genuine subgraph $H$ of $G$ (maximality). From (c) follows the one to one correspondence between $\mathscr{C}$ and $\left(\mathscr{C}_{1}, \mathscr{C}_{2}\right)$. $\mathscr{C}_{1}$ resp. $\mathscr{C}_{2}$ have $h_{G / H}=h_{G}-h_{H}$ resp. $h_{H}$ elements by assumption. Therefore, $\mathscr{C}$ has $h_{G}$ elements.

(e) Let $G_{1}$ be one component of $G$, then $G^{\prime}=G-\left\{\sigma\left(G_{1}\right)\right\}$ is connected and $\mathscr{C}-\left\{G_{1}\right\}$ is a maximal forest for $G^{\prime}$. Proceeding this way we finally have removed $h_{G}$ lines and get a connected graph which must be a tree.

(f) Let $\underline{\alpha}=\left(\alpha_{1}, \ldots, \alpha_{L}\right)$ be a point satisfying $\alpha_{\ell} \geqq 0$ and let us relabel the lines of $G$ such that $\alpha_{1} \leqq \alpha_{2} \ldots \leqq \alpha_{L}$. Let $G_{j}$ be the graph consisting of lines $\ell_{1}, \ldots, \ell_{j}$ and all their endpoints $\left(G_{0}=\emptyset\right)$. For each $k\left(1 \leqq k \leqq h_{G}\right)$ there is a unique $j(k)$ with $h_{G_{j(k)}}=k, h_{G_{j(k)-1}}=k-1$. Let $H_{k}$ be that component of $G_{j(k)}$ which contains line $\ell_{j(k)}$. Then $(\mathscr{C}, \sigma)$ with $\mathscr{C}=\left\{H_{k}, k=1, \ldots, h_{G}\right\}$ and $\sigma\left(H_{k}\right)=\ell_{j(k)}$ is a labelled forest and $\left(\alpha_{1}, \ldots, \alpha_{L}\right) \in \mathscr{D}(\mathscr{C}, \sigma)$.

(g) Let $(\mathscr{C}, \sigma) \neq\left(\mathscr{C}^{\prime}, \sigma^{\prime}\right)$ and $\alpha \in \mathscr{D}(\mathscr{C}, \sigma) \cap \mathscr{D}\left(\mathscr{C}^{\prime}, \sigma^{\prime}\right)$. We will show that $\alpha_{\ell}=\alpha_{\ell^{\prime}}$ for $\ell \neq \ell^{\prime}$. The set of such $\underline{\alpha}$ has Lebesgue measure zero. Since both $\mathscr{C}$ and $\mathscr{C}^{\prime}$ contain all components $G_{i}$ of $G$ there must exist a subgraph $H \in \mathscr{C} \cap \mathscr{C}^{\prime}$ for which $\sigma(H) \neq \sigma^{\prime}(H)$ and hence $\alpha_{\sigma(H)}=\alpha_{\sigma^{\prime}(H)}$.

Let $G$ be a connected graph and $\mathscr{C}$ a family of non-trivial non-overlapping subgraphs of $G$. The definition of $\mathscr{M}(H)$ and the concept of a labelled family $(\mathscr{C}, \sigma)$ can obviously be extended to this situation. The elements $H \in \mathscr{C}$ need not be 1 PI (not even connected) nor need $\mathscr{C}$ be maximal. We require, however, that $G \in \mathscr{C}$ and that for every $H \in \mathscr{C} H / \mathscr{M}(H)$ consists of a connected graph $\bar{H}$ plus possibly some trivial graphs not connected to $\bar{H}$. Given any $\mathscr{C}$ these requirements can always be satisfied by adding some suitable subgraphs to $\mathscr{C}$.

To any vertex $V_{a}$ of $\bar{H}(H \in \mathscr{C})$ we assign a momentum $q_{a}$ which is the sum of all those $p_{i}$ associated with vertices $V_{i}$ which are mapped to $V_{a}$ by $H \rightarrow H / \mathscr{M}(H)$. Let $q_{H}, H \in \mathscr{C}$ be the momenta associated with all but one of the vertices of $\bar{H}$. The new momenta $g=\left(q_{H}, H \in \mathscr{C}\right)$ are obtained from $p=\left(p_{i}, i=1, \ldots, M-1\right)$ by a linear transformation $R: q=R p$. In order to express $V(p, \underline{u}, \underline{\alpha})$ in terms of $g$, we decompose $\underline{u}=\left(u_{\ell}, \ell \in \mathscr{L}_{G}\right)$ and the transformed incidence matrix $e R^{+}$in a similar way: $\underline{u}=\left(\underline{u}_{H}=\left(u_{\ell}, \ell \in \mathscr{L}_{\vec{H}}\right), H \in \mathscr{C}\right), e R^{+}=\left(e_{H H^{\prime}}, H, H^{\prime} \in \mathscr{C}\right)$. If we introduce in addition scaled quantities $\tilde{q}=\left(\tilde{q}_{H}=q_{H} \zeta_{H}, H \in \mathscr{C}\right), \underline{\tilde{u}}=\left(\tilde{u}_{H}=\underline{u}_{H} / \zeta_{H}, H \in \mathscr{C}\right)$ and

$$
\tilde{e}=\left(\tilde{e}_{H H^{\prime}}=e_{H H^{\prime}} \zeta_{H^{\prime}} / \zeta_{H}, H, H^{\prime} \in \mathscr{C}\right)
$$


we find $V(\underline{p}, \underline{u}, \underline{\alpha})=\left(\tilde{q}^{+}, \underline{\tilde{u}}^{+}\right) \tilde{M}^{-1}\left(\begin{array}{l}\underline{\tilde{q}} \\ \underline{\tilde{u}}\end{array}\right)$ with $\tilde{M}=\left(\begin{array}{cc}0 & -2 \tilde{e}^{+} \\ -2 \tilde{e} & -4 \beta\end{array}\right)$ where $\beta$ is the diagonal matrix with elements $\beta_{\ell}=\alpha_{\ell} / \zeta_{H}^{2}, \ell \in \mathscr{L}_{\bar{H}}, H \in \mathscr{C}$.

Lemma 4. (a) $\operatorname{det} R= \pm 1$;

(b) $e R^{+}$has the following block structure

$e_{H H^{\prime}}\left\{\begin{array}{l}=0 \text { if } H \supset H^{\prime} ; \\ \text { is the incidence matrix } e_{\bar{H}} \text { for } \bar{H} \text { if } H=H^{\prime} ; \\ \text { indicates how } H^{\prime} \text { is contained in } H \text { if } H \supsetneq H^{\prime} ;\end{array}\right.$

(c) $d(\alpha)=\tilde{d} \prod_{H \in \mathscr{C}} \zeta_{H}^{2 h_{\bar{H}}}=\tilde{d} \prod_{H \in \mathscr{C}} t_{H}^{2 h_{H}}$ and $\tilde{d}=\operatorname{det} \tilde{M} /(-4)^{L} \geqq \prod_{H \in \mathscr{C}} \tilde{d}_{H}$ as long as $\beta_{\ell} \geqq 0$ for all $\ell \in \mathscr{L}_{G}$ where

$$
\tilde{d}_{H}=\operatorname{det}\left(\begin{array}{cc}
0 & -2 \tilde{e}_{H H}^{+} \\
-2 \tilde{e}_{H H} & -4 \beta_{H}
\end{array}\right) /(-4)^{\left|\mathscr{L}_{\bar{H}}\right|} ;
$$

(d) considered as functions of $(t, \beta)$ the matrix elements of $\tilde{M}$ are independent of $t_{G}$ and polynomials in the remaining variables;

(e) if $\mathscr{C}$ is such that $h_{\overline{\mathrm{H}}} \leqq 1$ for all $H \in \mathscr{C}$, and $\zeta_{H}$ are scaling variables for $(\mathscr{C}, \sigma)$, then $\tilde{d} \geqq 1$ and therefore $\tilde{d}^{-1}$ and $\tilde{M}^{-1}$ are $C^{\infty}$ in $(t, \beta)$ from the domain $\mathscr{D}(\mathscr{C}, \sigma)$. This is true in particular if $G$ is $1 P I$ and $(\mathscr{C}, \sigma)$ is a labelled forest for $G$.

Proof. (a) Up to permutations $R$ is a triangular matrix with all diagonal elements $=1$;

(b) Introducing internal momenta $\underline{k}=\left(k_{\ell}, \ell \in \mathscr{L}_{G}\right)$ flowing through all lines, momentum conservation at each vertex is expressed by $\underline{p}+e^{+} \underline{k}=0$. The block structure follows immediately from $\underline{q}+\operatorname{Re}^{+} \underline{k}=0$;

(c) The factorization follows from a), $\left|\mathscr{L}_{\bar{H}}\right|+1=\left|\mathscr{V}_{\bar{H}}\right|+h_{\bar{H}}$ and the relation between $\zeta$ and $t$. The inequality is due to the fact that every pair of trees for $G / H$ and $H$ corresponds to a unique tree for $G$ and that the contribution of each tree to $d(\alpha)$ is non-negative;

(d) The matrix elements of $\tilde{e}$ are polynomials due to b) and clearly both $\tilde{e}$ and $\beta$ are independent of $t_{G}$;

(e) $\tilde{d}_{H}=1$ if $h_{\bar{H}}=0$ and $\tilde{d}_{H}=\sum_{\ell \in \mathscr{L}_{\bar{H}}} \beta_{\ell}$ if $h_{\bar{H}}=1 . \beta_{\sigma(H)}=1$ and therefore $\tilde{d}_{H} \geqq 1$. In the domain $\mathscr{D}(\mathscr{C}, \sigma) \beta_{\ell} \leqq 1$ for all $\ell$ and $t_{H} \leqq 1$ for $H \neq G$. The statements follow from these results and $(\mathrm{d})$.

Remark. We may consider a family $\mathscr{C}^{\prime}$ obtained from $\mathscr{C}$ by omission of one element $H_{0} \in \mathscr{M}\left(H_{1}\right)$ and build momenta $q_{H}^{\prime}, H \in \mathscr{C}^{\prime}$ for this new family. Obviously there is a choice for the $q_{H}$ and $q_{H}^{\prime}$ (i.e. choice for the vertex whose momentum is omitted from $\left.q_{H}\right)$ such that $\underline{q}_{H_{1}}^{\prime}=\left(\underline{q}_{H_{1}}, \underline{q}_{H_{0}}\right)$ and $\underline{q}_{H}^{\prime}=\underline{q}_{H}$ for all other $H \in \mathscr{C}^{\prime}$.

\section{Appendix B}

Let $G$ be $1 \mathrm{PI},(\mathscr{C}, \sigma)$ a labelled forest for $G$ and $H \in \mathscr{C}(H \neq G)$. Corresponding to $(\mathscr{C}, \sigma)$ there is a unique pair of labelled forests $\left(\left(\mathscr{C}_{1}, \sigma_{1}\right),\left(\mathscr{C}_{2}, \sigma_{2}\right)\right)$ for $G / H$ and $H$ [Lemma 3 (c)]. 
We want to relate the contributions of these three labelled forests to $\mathscr{T}_{G}, \mathscr{T}_{G / H}$ and $\mathscr{T}_{H}$ respectively. We will prove that the singular part of the $t_{H}$-integral [as defined by Eq. (III.5)] in $\mathscr{T}_{H}$ is a polynomial $P\left(q_{H}\right)$ of degree $\omega_{H}$ in the external momenta $g_{H}$ of $H$ (a homogeneous polynomial of degree $\omega_{H}$ in $g_{H}$, masses and $\sqrt{\varepsilon}$ if all spin polynomials $Z_{\ell}$ are homogeneous in $-i \partial / \partial u$, masses and $\sqrt{\varepsilon}$ ). On the other hand we will express the singular part of the $t_{H}$-integral in $\mathscr{T}_{G}$ in terms of the same polynomial $P\left(g_{H}\right)$ inserted as vertex function into $\mathscr{T}_{G / H}$.

We will show all this for the regularized amplitudes $\mathscr{T}$. The results apply as well if we first perform subtractions for some subgraphs $H^{\prime} \in \mathscr{C}, H^{\prime} \downarrow H$, because the subtracted amplitudes are sums of regularized amplitudes for various reduced graphs, each of them with the above mentioned properties. In particular reducing a subgraph $H^{\prime}$ to a vertex with vertex function $P\left(g_{H^{\prime}}\right)$ which is a polynomial of degree $\omega_{H^{\prime}}$ does not change the degree of divergence $\omega_{H}$ for any $H \supset H^{\prime}$.

Let us first consider the $t_{G}$-integral in Equation (III.4)

$$
\int_{0}^{\infty}\left(d t_{G} / t_{G}\right) t_{G}^{\nu h_{G}-\omega_{G}} g_{\varepsilon}(t, \underline{\beta}, \underline{q})
$$

Assuming that all $Z_{\ell}$ are homogeneous the entire dependence of $g_{\varepsilon}$ on $t_{G}, q$, masses and $\varepsilon$ comes from the combinations $t_{G} q, t_{G} m_{\ell}$ and $t_{G} \sqrt{\varepsilon}$. First of all there is no singular part as long as $\omega_{G}<0$. If $\omega_{G} \geqq 0$ the singular part is proportional to $\left[\left(\omega_{G} !\right)^{-1}\left(d / d t_{G}\right)^{\omega_{G}} g_{\varepsilon}\right]_{t_{G}=0}$ which certainly is a homogeneous polynomial of degree $\omega_{G}$.

In order to study the singular part of the $t_{H}$-integrals we replace the maximal forest $\mathscr{C}$ by a family $\mathscr{C}=\{G, H\}$ and (using the notation of Lemma 4) introduce momenta $g=\left(q_{G}, q_{H}\right)$ correspondingly. Inserting the polynomial $P\left(g_{H}\right)$ as vertex into the amplitude for $G / H$ amounts to the substitution $U_{H}: q_{H} \rightarrow q_{H}-i e_{G H}^{+} \partial / \partial \underline{u}_{G}^{+}$ and acting with the differential operator $U_{H}\left(P\left(g_{H}\right)\right)$ on the integrand $I_{G / H}$; the external momenta of $H$ differ from the external momenta of $G$ associated with vertices of $H$ by linear combinations of internal momenta of $G / H$.

Apart from factors and integrals common to both amplitudes, the expressions for $\mathscr{T}_{G}$ resp. $\mathscr{T}_{G / \boldsymbol{H}}$ (with the insertion corresponding to $H$ ) are

$$
\left.\xi_{H}^{v h_{H}} \int_{0}^{1}\left(d t_{H} / t_{H}\right) t_{H}^{\nu h_{H}-\omega_{H}} I_{G}\right|_{\underline{\underline{u}}_{H}=0} ; I_{G}=Z_{H}\left(-i \partial / \partial \underline{\tilde{u}}_{H}\right)(\operatorname{det} \tilde{M})^{-n / 2} \exp i W
$$

resp.

$$
U_{H}\left(\xi_{H}^{\omega_{H}} P\left(q_{H}\right)\right) I_{G / H} ; I_{G / H}=\left(\operatorname{det} \tilde{M}_{G / H}\right)^{-n / 2} \exp i W_{G / H} .
$$

Using the homogeneity of $Z_{H}$ and $P\left(g_{H}\right)$ we can compensate the difference in scaling variables used for $\mathscr{T}_{G}$ and $\mathscr{T}_{H}$ and find

$$
\xi_{H}^{\omega_{H}} P\left(q_{H}\right)=\left\{\left(\omega_{H} !\right)^{-1}\left(d / d t_{H}\right)^{\omega_{H}}\left[Z_{H}\left(-i \partial / \partial \underline{\tilde{u}}_{H}\right)\left(\operatorname{det} \tilde{M}_{H}\right)^{-n / 2} \exp i W_{H}\right]_{\tilde{\underline{u}}_{H}=0}\right\}_{t_{H}=0}
$$

with (X stands for either $G / H$ or $H) \tilde{M}=\hat{M}-2 t_{H}\left(\hat{e}+\hat{e}^{+}\right)$;

$$
\begin{aligned}
& \hat{M}=\left(\begin{array}{cc}
\tilde{M}_{G / H} & 0 \\
0 & \tilde{M}_{H}
\end{array}\right) ; \quad \tilde{M}_{X}=\left(\begin{array}{cc}
0 & -2 e_{X}^{+} \\
-2 e_{X} & -4 \beta_{X}
\end{array}\right) ; \quad \hat{e}=\left(\begin{array}{c}
0\left(\begin{array}{cc}
0 & 0 \\
e_{G H} & 0
\end{array}\right) \\
0 \\
0
\end{array}\right) ; \\
& W=Q^{+} \tilde{M}^{-1} Q-m_{G / H}^{2}-m_{H}^{2} ; W_{X}=Q_{X}^{+} \tilde{M}_{X}^{-1} Q_{X}-m_{X}^{2} ; Q=\left(\begin{array}{c}
Q_{G / H} \\
Q_{H}
\end{array}\right) ; Q_{X}=\left(\begin{array}{l}
\tilde{g}_{X} \\
\tilde{\tilde{u}}_{X}
\end{array}\right) ;
\end{aligned}
$$


and

$$
m_{X}^{2}=\sum_{\ell \in \mathscr{L}_{X}} \alpha_{\ell}\left(m_{\ell}^{2}-i \varepsilon\right)
$$

Lemma 5. $U_{H}\left[\xi_{H}^{\omega_{H}} P\left(q_{H}\right)\right] I_{G / H}=\left[\left(\omega_{H} !\right)^{-1}\left(d / d t_{H}\right)^{\omega_{H}} I_{G}\right]_{t_{H}=\underline{\tilde{u}}_{H}=0}$.

Remark. This establishes the required connection between the singular part of the $t_{H}$-integrals in $\mathscr{T}_{G}$ and $\mathscr{T}_{H}$. The remaining difference, a factor $\xi_{H}^{v h_{H}}$, is the origin of $\sum_{m} c_{m} m^{-1} \xi_{H}^{v m}$ in Equation (III.14), each term $\xi_{H}^{v m}$ arises from reduced graphs of $H$ with $m$ loops.

Proof. First we note that $\left.(\operatorname{det} \tilde{M})\right|_{t_{H}=0}=\left(\operatorname{det} \tilde{M}_{G / H}\right)\left(\operatorname{det} \tilde{M}_{H}\right),\left.W\right|_{t_{H}=0}=W_{G / H}+$ $\left.W_{H}\right|_{t_{H}=0}$ and $\tilde{q}_{H}=\xi_{H} t_{H} q_{H}$. It is therefore sufficient to show that

$$
-i\left(d / d t_{H}\right)\left\{(\operatorname{det} \tilde{M})^{-n / 2} \exp i W\right\}=U_{H}\left(d W_{H} / d t_{H}\right)\left\{(\operatorname{det} \tilde{M})^{-n / 2} \exp i W\right\} .
$$

where $\left(d / d t_{H}\right)$ is taken either for fixed $\left(q_{H}, u_{H}\right)$ or fixed $\left(q_{H}, \tilde{u}_{H}\right)$. This can be done, using $U_{H}(Q)=Q-i t_{H} \hat{e}^{+} \partial / \partial Q^{+}$and some properties of the matrix $\hat{e}: \hat{e} \hat{M}^{-1} \hat{e}=0$, $\operatorname{Tr}\left(\hat{e} \hat{M}^{-1}\right)=0, \hat{e}^{+} \hat{M}^{-1} d Q / d t_{H}=0$. Once again we need $\partial u_{\ell^{\prime}} / \partial u_{\ell}=\delta_{\ell \ell^{\prime}} g_{\mu \mu}=\delta_{\ell \ell^{\prime}} n$ and it is therefore important that we interprete $\underline{u}$ as an element of the abstract algebra of " $n$-dimensional" covariants.

\section{Appendix C. Completion of the Proof of Theorem 2}

The problem to be considered is that there may be subgraphs $H$ of $G$ which have two components $H_{l}$ and $H_{r}$ joined by line $\ell$. These become 1 PI by contraction of line $\ell$. The forests used to renormalize $\mathscr{T}_{G}$ may contain $\left\{H_{1}, H_{r}\right\}$ whereas the forests for $\mathscr{T}_{G / \ell}$ may contain $\{H\},\left\{H, H_{l}\right\}$ or $\left\{H, H_{r}\right\}$. We shall recursively remove the latter forests from $\mathscr{F}_{G / \ell}$ and replace them by forests (for $G$ ) containing $\left\{H_{l}, H_{r}\right\}$. We show that $\Sigma \Pi\left(-C_{H}\right)$ does not change under these replacements due to the factorization $I_{H}=I_{H_{l}} I_{H_{r}}$ and the identity

$$
\left(-C_{H}+C_{H} C_{H_{l}}+C_{H} C_{H_{r}}\right)=C_{H_{l}} C_{H_{r}} .
$$

Define

$$
\begin{aligned}
\mathscr{H}_{K}= & \left\{H \subset G: \ell \notin H ; H=H_{l} \cup H_{r} ; V_{i_{\ell}} \in \mathscr{V}_{H_{1}} ; V_{f_{\ell}} \in \mathscr{V}_{H_{r}} ; h_{H}=K ; H_{l} \cap H_{r}=\emptyset ;\right. \\
& \left.H_{l}, H_{r} \text { are } 1 \mathrm{PI}\right\} .
\end{aligned}
$$

Since there is a one to one correspondence between subgraphs of $G / \ell$ and those of $G$ not containing line $\ell$, we may consider these subgraphs also to be in $G / \ell$.

Put $\mathscr{F}=\{$ forests for $G$ which do not contain subgraphs of the type shown in Figure 1\},

$$
\mathscr{F}^{\ell}=\{\text { forests for } G / \ell\}, \quad \mathscr{F}_{1}=\left\{F \in \mathscr{F}^{\ell}: F \ni H 1 \mathrm{PI} \text { in } G / \ell\right\} .
$$


$\mathscr{F}_{K+1}$ is constructed in the following way from $\mathscr{F}_{K}$ : For all $H \in \mathscr{H}_{K}$ replace all forests which contain either $\{H\}$ or $\left\{H, H_{l}\right\}$ or $\left\{H, H_{r}\right\}$ by corresponding forests which contain $\left\{H_{l}, H_{r}\right\}$. This procedure gives

$\mathscr{F}_{K}=\left\{F \in \mathscr{F}\right.$ : If $H \in F$, then either $H$ is $1 \mathrm{PI}$ in $G$ or $H$ is $1 \mathrm{PI}$ in $G / \ell$ and $h_{H}>K$; for any pair $\left\{H_{1}, H_{2}\right\} \in F$ either $H_{1} \otimes H_{2}$ in $G / \ell$ or $\left.H_{1} \cup H_{2} \in \bigcup_{K^{\prime} \leqq K} \mathscr{H}_{K^{\prime}}\right\}$.

We find $\mathscr{F}_{h_{G}}=\{F \in \mathscr{F}: F \ni H 1 \mathrm{PI}$ in $G\}$.

We prove now the

Proposition 4. If (1) holds for all $H \in \oplus \mathscr{H}_{K}$, then

$$
\sum_{F \in \mathscr{F}_{K}} \prod_{H \in F}\left(-C_{H}\right)=\sum_{F \in \mathscr{F}_{K+1}} \prod_{H \in F}\left(-C_{H}\right) \quad \text { for } \quad 1 \leqq K<h_{G} .
$$

Proof. We observe the following: Let $\left\{H^{1}, H^{2}\right\} \subset \mathscr{H}_{\mathrm{K}+1}$, then

i) $H^{1} \circ H^{2}$ and, therefore, there is no $F \in \mathscr{F}_{K}$ with $\left\{H^{1}, H^{2}\right\} \in F$;

ii) $H_{l}^{1} \circ H_{l}^{2}$ or $H_{r}^{1} \circ H_{r}^{2}$ or $\max \left\{h_{H_{l}^{1} \cup H_{r}^{2}}, h_{H_{l}^{2} \cup H_{r}^{r}}\right\}>K+1$ and, therefore, there is no $F \in \mathscr{F}_{K+1}$ with $\left\{H_{l}^{1}, H_{r}^{1}, H_{l}^{2}, H_{r}^{2}\right\} \subset F$.

That means that we can uniquely decompose $(\oplus=$ disjoint union)

$$
\begin{aligned}
\mathscr{F}_{K} & \left.=\bigoplus_{H \in \mathscr{H}_{K+1}} \bigoplus_{F \in \mathscr{F}_{H}}\left\{F \oplus\{H\}, F \oplus\left\{H, H_{l}\right\}, F \oplus\left\{H, H_{r}\right\}\right\}\right) \oplus \hat{\mathscr{F}}_{K} ; \\
\mathscr{F}_{K+1} & \left.=\bigoplus_{H \in \mathscr{H}_{K+1}} \bigoplus_{F \in \mathscr{F}_{H}}\left\{F \oplus\left\{H_{l}, H_{r}\right\}\right\}\right) \oplus \hat{\mathscr{F}}_{K} ;
\end{aligned}
$$

where $\hat{\mathscr{F}}_{K}=\mathscr{F}_{K} \cap \mathscr{F}_{K+1}$, and

$$
\mathscr{F}^{H}=\left\{F \in \hat{\mathscr{F}}_{\mathrm{K}}: H^{\prime} \in F \Rightarrow H^{\prime} \text { non-overlapping with } H, H_{l}, H_{r}\right\} .
$$

Using this decomposition and Equation (1), Equation (2) is immediately proved. We get

$$
\sum_{F \in \mathscr{F}_{1}} \prod_{H \in F}\left(-C_{H}\right) \mathscr{T}_{G / \ell}=\sum_{F \in \mathscr{F}_{h_{G}}} \prod_{H \in F}\left(-C_{H}\right) \mathscr{T}_{G / \ell}
$$

and this completes the proof of Theorem 2 .

\section{References}

1. t'Hooft, G., Veltman, M.: Nucl. Phys. B 44, 189 (1972)

2. Bollini, C. G., Giambiagi, J. J.: Phys. Letters 40 B, 566 (1972)

Cicuta, G. M., Montaldi, E.: Nuovo Cimento Letters 4, 329 (1972)

3. Becchi,C., Rouet,A., Stora,R.: Broken Symmetries: Perturbation theory of renomalizable models. Lectures given at the International School of Elementary Particle Physics, Basco Polje, 1974

4. t'Hooft, G., Veltman, M.: Diagrammar, CERN Report 73/9

5. Hepp, K.: Renormalization theory in statistical mechanics and quantum field theory (Les Houches 1970), (C. de Witt, R. Stora eds.). New York: Gordon and Breach 1971

6. Speer, E. R.: J. Math. Phys. 15, 1 (1974)

7. Collins, J.C.: Nucl. Phys. B 92, 477 (1975)

8. Lam, Y.M.P.: Phys. Rev. D 6, 2145 (1972)

9. Speer,E.R.: Generalized Feynman amplitudes. Princeton: Princeton University Press 1969 
10. Speer,E.R., Westwater, M. J.: Ann. Inst. H. Poincaré 14 A, 1 (1971)

11. Lowenstein, J.H.: Commun. math. Phys. 24, 1 (1971)

12. Gelfand, I. M., Shilov, G.E.: Generalized functions, Vol. I. New York: Academic Press 1964

13. Bollini, C. G., Giambiagi, J. J.: Acta Phys. Acustriaca 38, 211 (1973)

14. Zimmermann, W.: Commun. math. Phys. 15, 208 (1969)

15. Lowenstein, J.H.: Seminars on Renormalization Theory, Vol. I., Maryland Technical Report 73-068 (1972)

16. Gomez, M., Lowenstein, J.H.: Phys. Rev. D7, 550 (1973)

17. Nakanishi, N.: Graph theory and Feynman integrals. New York: Gordon and Breach 1971

Communicated by K. Symanzik

Received July 4, 1975; in revised form April 7, 1976 